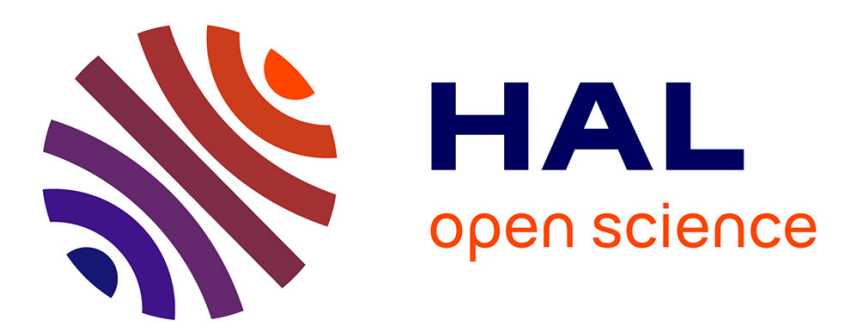

\title{
A posteriori error estimates via equilibrated stress reconstructions for contact problems approximated by Nitsche's method
}

\author{
Daniele Antonio Di Pietro, Ilaria Fontana, Kyrylo Kazymyrenko
}

\section{To cite this version:}

Daniele Antonio Di Pietro, Ilaria Fontana, Kyrylo Kazymyrenko. A posteriori error estimates via equilibrated stress reconstructions for contact problems approximated by Nitsche's method. Computers \& Mathematics with Applications, 2022, 111, pp.61-80. 10.1016/j.camwa.2022.02.008 . hal-03354078

\author{
HAL Id: hal-03354078 \\ https://hal.science/hal-03354078
}

Submitted on 24 Sep 2021

HAL is a multi-disciplinary open access archive for the deposit and dissemination of scientific research documents, whether they are published or not. The documents may come from teaching and research institutions in France or abroad, or from public or private research centers.
L'archive ouverte pluridisciplinaire $\mathbf{H A L}$, est destinée au dépôt et à la diffusion de documents scientifiques de niveau recherche, publiés ou non, émanant des établissements d'enseignement et de recherche français ou étrangers, des laboratoires publics ou privés. 


\title{
A posteriori error estimates via equilibrated stress reconstructions for contact problems approximated by Nitsche's method
}

\author{
Daniele A. Di Pietro ${ }^{1}$, Ilaria Fontana ${ }^{*, 1,2}$, and Kyrylo Kazymyrenko ${ }^{2}$ \\ ${ }^{1}$ IMAG, Univ Montpellier, CNRS, Montpellier, France \\ ${ }^{2}$ IMSIA, UMR EDF-CNRS-CEA-ENSTA 9219, Palaiseau, France
}

\begin{abstract}
We present an a posteriori error estimate based on equilibrated stress reconstructions for the finite element approximation of a unilateral contact problem with weak enforcement of the contact conditions. We start by proving a guaranteed upper bound for the dual norm of the residual. This norm is shown to control the natural energy norm up to a boundary term, which can be removed under a saturation assumption. The basic estimate is then refined to distinguish the different components of the error, and is used as a starting point to design an algorithm including adaptive stopping criteria for the nonlinear solver and automatic tuning of a regularization parameter. We then discuss an actual way of computing the stress reconstruction based on the Arnold-Falk-Winther finite elements. Finally, after briefly discussing the efficiency of our estimators, we showcase their performance on a panel of numerical tests.
\end{abstract}

Keywords: unilateral contact problem, weakly enforced contact conditions, a posteriori error estimate, equilibrated stress reconstruction, Arnold-Falk-Winther mixed finite element, adaptivity

MSC2020 classification: $74 \mathrm{M} 15,74 \mathrm{~S} 05,65 \mathrm{~N} 15,65 \mathrm{~N} 30,65 \mathrm{~N} 50$

\section{Introduction}

In various fields of solid mechanics and engineering it is essential to describe contact and friction between two bodies. This is the case, e.g., when modelling foundations and joints in buildings or when considering impact problems. In this paper, we focus on a simplified unilateral contact problem without friction, for which contact is mathematically expressed by some inequalities and complementarity conditions. These constraints translate non-penetration as well as the absence of cohesive forces and friction between the two bodies. In order to deal with the above constraints from a numerical point of view, different strategies have been proposed in the literature, including penalized formulations, mixed formulations, and weak enforcement à la Nitsche. We focus on the latter, which does not require the introduction of Lagrange multipliers and results in a coercive formulation that is easy to implement. The literature on the numerical approximation of contact problems is vast, and a comprehensive state-of-the-art lies outside of the scope of the present papers. We refer to the review articles [33,9] and references therein for a broader discussion.

*Corresponding author 
Nitsche's method was originally introduced in [27] to weakly enforce Dirichlet boundary conditions. Its application to the unilateral contact problem considered in this paper was originally proposed in [11], where the well-posedness and convergence of a conforming finite element scheme are studied. Further extensions to problems involving friction or multiple bodies can be found in [12, 7, 29, 23]; we also mention [8] concerning the adaptation of these ideas to Hybrid High-Order discretizations [18, 17, 15]. A residual-based a posteriori error analysis can be found in [10] based on a saturation assumption.

In this paper we follow a different path and carry out an a posteriori error analysis based on equilibrated tractions in the spirit of the Prager-Synge equality [28] (see also [21] and [32, Chapter 7]). This approach, which does not require the saturation assumption when the dual norm of the residual is considered as an error measure, has also the advantage of avoiding unknown constants in the upper bound. The corresponding error estimate can additionally be refined in order to distinguish the various components of the error (discretization, linearization, regularization). This decomposition is leveraged here to design a fully adaptive resolution algorithm including an a posteriori-based stopping criterion for the nonlinear solver and the automatic tuning of the regularization parameter.

A crucial ingredient of our a posteriori analysis is a novel $\mathbb{E}(\mathbf{d i v})$-conforming stress reconstruction obtained from the numerical solution by solving small problems on patches around mesh vertices. In the spirit of $[30,5]$, we use the Arnold-Falk-Winther mixed finite element spaces with weakly enforced symmetry [1]; strong symmetry could be enforced using the Arnold-Winther finite element spaces [2] as in [30], but would come at a significantly higher computational cost. The stress reconstruction is built so that its divergence and its normal component on the contact and Neumann portions of the domain boundary are locally in equilibrium with the volume and surface source terms, respectively (such equilibrium properties are not satisfied by stress fields resulting from $\boldsymbol{H}^{1}$-conforming finite element approximations). In order to distinguish the various error components, the stress reconstruction is additionally split so as to identify the contributions to the error resulting from linearization and regularization.

The main results of the paper are briefly summarized in what follows. The basic error estimate of Theorem 4 establishes a guaranteed upper bound for the dual norm of the residual. Such norm is shown in Theorem 7 to control the energy norm of the error up to a boundary term on the contact region (this term can be eliminated when a saturation assumption similar to the one used in [10] holds). A refined error estimate distinguishing the error components is derived in Theorem 11.

The rest of the paper is organized as follows. In Section 2 we describe the unilateral contact problem and its finite element approximation à la Nitsche. In Section 3 we derive a basic estimate for the dual norm of the residual and compare this norm with the energy norm. Section 4 contains a refined version of the estimate distinguishing the error components which serves as a starting point for the development of a fully adaptive algorithm. An equilibrated stress reconstruction based on the Arnold-Falk-Winther finite element is then proposed in Section 5. Section 6 briefly addresses the efficiency of the error estimators. Finally, some numerical results performed with the open source software FreeFem++ are presented in Section 7.

\section{Setting}

In this section we discuss the contact problem and its finite element discretization with weakly enforced contact conditions. 


\subsection{Unilateral contact problem}

\subsubsection{Strong formulation}

Let $d \in\{2,3\}$ and let $\Omega \subset \mathbb{R}^{d}$ be a connected open subset of $\mathbb{R}^{d}$ representing an elastic body. We suppose that $\Omega$ is a polygon (if $d=2$ ) or a polyhedron (if $d=3$ ), and that its boundary $\partial \Omega$ is partitioned into three non-overlapping parts $\Gamma_{D}, \Gamma_{N}$, and $\Gamma_{C}$ such that $\left|\Gamma_{D}\right|>0$ and $\left|\Gamma_{C}\right|>0$ $(|\cdot|$ denotes here the Hausdorff measure). In its reference configuration, the elastic body is in contact through $\Gamma_{C}$ with a rigid foundation, and we assume that the (unknown) contact zone in the deformed configuration will be included in $\Gamma_{C}$. The body is clamped at $\Gamma_{D}$ and it is subjected to a volume force $\boldsymbol{f} \in \boldsymbol{L}^{2}(\Omega)$ and to a surface load $\boldsymbol{g}_{N} \in \boldsymbol{L}^{2}\left(\Gamma_{N}\right)$ on $\Gamma_{N}$; see Figure 5 for an example.

Denoting by $\boldsymbol{n}$ the unit normal vector on $\partial \Omega$ pointing out of $\Omega$, for any displacement field $v: \Omega \rightarrow \mathbb{R}^{d}$ and for any density of surface forces $\sigma(v) n$ defined on $\partial \Omega$, we have the following (unique) decomposition into normal and tangential components:

$$
v=v^{n} n+v^{t} \quad \text { and } \quad \sigma(v) n=\sigma^{n}(v) n+\sigma^{t}(v) .
$$

We consider the following problem: Find the displacement field $\boldsymbol{u}: \Omega \rightarrow \mathbb{R}^{d}$ such that

$$
\begin{aligned}
\boldsymbol{d i v} \boldsymbol{\sigma}(\boldsymbol{u})+\boldsymbol{f} & =\mathbf{0} & & \text { in } \Omega, \\
\boldsymbol{\sigma}(\boldsymbol{u}) & =\boldsymbol{A} \boldsymbol{\varepsilon}(\boldsymbol{u}) & & \text { in } \Omega, \\
\boldsymbol{u} & =\mathbf{0} & & \text { on } \Gamma_{D}, \\
\boldsymbol{\sigma}(\boldsymbol{u}) \boldsymbol{n} & =\boldsymbol{g}_{N} & & \text { on } \Gamma_{N}, \\
u^{n} \leq 0, \sigma^{n}(\boldsymbol{u}) \leq 0, \sigma^{n}(\boldsymbol{u}) u^{n} & =0 & & \text { on } \Gamma_{C}, \\
\boldsymbol{\sigma}^{\boldsymbol{t}}(\boldsymbol{u}) & =\mathbf{0} & & \text { on } \Gamma_{C},
\end{aligned}
$$

where $\boldsymbol{\varepsilon}(\boldsymbol{v}):=\frac{1}{2}\left(\boldsymbol{\nabla} \boldsymbol{v}+\boldsymbol{\nabla} \boldsymbol{v}^{\top}\right)$ is the strain tensor field, $\boldsymbol{\sigma}(\boldsymbol{v}) \in \mathbb{R}_{\mathrm{sym}}^{d \times d}$ is the Cauchy stress tensor, div is the divergence operator acting row-wise on tensor valued functions, and $\boldsymbol{A}$ is the fourth order symmetric elasticity tensor such that, for all second-order tensor $\boldsymbol{\tau}, \boldsymbol{A} \boldsymbol{\tau}=\lambda \operatorname{tr}(\boldsymbol{\tau}) \boldsymbol{I}_{d}+2 \mu \boldsymbol{\tau}$, with $\lambda$ and $\mu$ denoting the Lamé parameters.

Remark 1 (Contact conditions). The first contact condition (2.2e) is a complementarity condition: if, at a point $\boldsymbol{x} \in \Gamma_{C}$, there is no contact in the deformed configuration (i.e., $u^{n}<0$ ), then the normal stress vanishes at that point (i.e., $\sigma^{n}(\boldsymbol{u})=0$ ); on the other hand, if at $\boldsymbol{x} \in \Gamma_{C}$ the normal stress is nonzero (i.e., $\sigma^{n}(\boldsymbol{u})<0$ ), then in the deformed configuration we still have contact in $\boldsymbol{x}$ (i.e., $u^{n}=0$ ). These conditions also account for the absence of normal cohesive forces between the elastic body and the rigid foundation. The second contact condition (2.2f) simply establishes the absence of friction on $\Gamma_{C}$.

The incorporation of standard friction models to the following a posteriori theory seems possible, but lies outside of the scope of the present paper. This subject will be addressed in future works.

\subsubsection{Weak formulation}

Let $D$ denote a measurable set of $\mathbb{R}^{d}$. In what follows, $D$ will be typically either equal to $\Omega$ or to the union of a finite subset of mesh elements. We denote by $H^{s}(D), s \in \mathbb{R}$, the usual Sobolev space of index $s$ on $D$, with the convention that $H^{0}(D):=L^{2}(D)$, the space of square-integrable functions on $D$. Its vector and tensor versions are denoted respectively by $\boldsymbol{H}^{s}(D):=\left[H^{s}(D)\right]^{d}$ and $\mathbb{H}^{s}(D):=\left[H^{s}(D)\right]^{d \times d}$. We let, similarly, $\boldsymbol{L}^{2}(D):=\left[L^{2}(D)\right]^{d}$ and $\mathbb{L}^{2}(D):=\left[L^{2}(D)\right]^{d \times d}$. Moreover, $\|\cdot\|_{s, D}$ denotes the norm of $H^{s}(D)$ or $\boldsymbol{H}^{s}(D)$ according to its argument. The first 
subscript is omitted when $s=0$, i.e., $\|\cdot\|_{D}$ is the standard norm of $L^{2}(D), L^{2}(D)$, or $\mathbb{L}^{2}(D)$ according to its argument. The usual inner products of these spaces are denoted by $(\cdot, \cdot)_{D}$, with the convention that the subscript is omitted when $D=\Omega$. In what follows, we will also need the space $\mathbb{H}(\mathbf{d i v}, D)$ spanned by functions of $\mathbb{L}^{2}(D)$ with weak (row-wise) divergence in $\boldsymbol{L}^{2}(D)$.

Denote by $\boldsymbol{H}_{D}^{1}(\Omega)$ the subspace of $\boldsymbol{H}^{1}(\Omega)$ incorporating the Dirichlet boundary condition on $\Gamma_{D}$, and by $\boldsymbol{K}$ its subset spanned by admissible displacements, i.e.,

$$
\boldsymbol{H}_{D}^{1}(\Omega):=\left\{\boldsymbol{v} \in \boldsymbol{H}^{1}(\Omega): \boldsymbol{v}=\mathbf{0} \text { on } \Gamma_{D}\right\}, \quad \boldsymbol{K}:=\left\{\boldsymbol{v} \in \boldsymbol{H}_{D}^{1}(\Omega): v^{n} \leq 0 \text { on } \Gamma_{C}\right\} .
$$

The weak formulation of problem (2.2) corresponds to the following variational inequality (see, e.g., [24]): Find $\boldsymbol{u} \in \boldsymbol{K}$ such that

$$
a(\boldsymbol{u}, \boldsymbol{v}-\boldsymbol{u}) \geq L(\boldsymbol{v}-\boldsymbol{u}) \quad \forall \boldsymbol{v} \in \boldsymbol{K},
$$

where the bilinear form $a: \boldsymbol{H}^{1}(\Omega) \times \boldsymbol{H}^{1}(\Omega) \rightarrow \mathbb{R}$ and the linear form $L: \boldsymbol{H}^{1}(\Omega) \rightarrow \mathbb{R}$ are defined as follows: For all $(\boldsymbol{u}, \boldsymbol{v}) \in \boldsymbol{H}^{1}(\Omega) \times \boldsymbol{H}^{1}(\Omega)$,

$$
a(\boldsymbol{u}, \boldsymbol{v}):=(\boldsymbol{\sigma}(\boldsymbol{u}), \boldsymbol{\varepsilon}(\boldsymbol{v})), \quad L(\boldsymbol{v}):=(f, \boldsymbol{v})+\left(\boldsymbol{g}_{N}, \boldsymbol{v}\right)_{\Gamma_{N}} .
$$

Problem (2.3) admits a unique solution by the Stampacchia theorem.

\subsection{Discretization}

Let $\left\{\mathcal{T}_{h}\right\}_{h}$ be a family of conforming triangulations of $\Omega$, indexed by the mesh size $h:=\max _{T \in \mathcal{T}_{h}} h_{T}$, where $h_{T}$ is the diameter of the element $T$. This family is assumed to be regular in the classical sense; see, e.g., [13, Eq. (3.1.43)]. Furthermore, each triangulation is conformal to the subdivision of the boundary into $\Gamma_{D}, \Gamma_{N}$, and $\Gamma_{C}$ in the sense that the interior of a boundary edge (if $d=2$ ) or face (if $d=3$ ) cannot have non-empty intersection with more than one part of the subdivision. Mesh-related notations that will be used in the a posteriori error analysis are collected in Table 1. For the sake of simplicity, from this point on we adopt the three-dimensional terminology and speak of faces instead of edges also in dimension $d=2$.

For any $X \in \mathcal{T}_{h} \cup \mathcal{F}_{h}$ mesh element or face, $\mathcal{P}^{n}(X)$ denotes the restriction to $X$ of $d$-variate polynomials of total degree $\leq n$, and we set $\mathcal{P}^{n}(X):=\left[\mathcal{P}^{n}(X)\right]^{d}$ and $\mathbb{P}^{n}(X):=\left[\mathcal{P}^{n}(X)\right]^{d \times d}$. We seek the displacement in the standard Lagrange finite element space of degree $p \geq 1$ with strongly enforced boundary condition on $\Gamma_{D}$ :

$$
\boldsymbol{V}_{h}:=\left\{\boldsymbol{v}_{h} \in \boldsymbol{H}_{D}^{1}(\Omega):\left.\boldsymbol{v}_{h}\right|_{T} \in \mathcal{P}^{p}(T) \text { for any } T \in \mathcal{T}_{h}\right\} .
$$

Denote by $[\cdot]_{\mathbb{R}^{-}}: \mathbb{R} \rightarrow \mathbb{R}^{-}$the projection on the half-line of negative real numbers $\mathbb{R}^{-}$, i.e., $[x]_{\mathbb{R}^{-}}:=\frac{1}{2}(x-|x|)$ for all $x \in \mathbb{R}$. For every real number $\theta$ and every positive bounded function $\gamma: \Gamma_{C} \rightarrow \mathbb{R}^{+}$, we define the following linear operator [9]:

$$
\begin{aligned}
P_{\theta, \gamma}^{n}: \boldsymbol{W} & \rightarrow L^{2}\left(\Gamma_{C}\right) \\
\boldsymbol{v} & \mapsto \theta \sigma^{n}(\boldsymbol{v})-\gamma v^{n},
\end{aligned}
$$

where $\boldsymbol{W}:=\left\{\boldsymbol{v} \in \boldsymbol{H}^{1}(\Omega):\left.\boldsymbol{\sigma}(\boldsymbol{v}) \boldsymbol{n}\right|_{\Gamma_{C}} \in \boldsymbol{L}^{2}\left(\Gamma_{C}\right)\right\}$ (notice that $\boldsymbol{V}_{h} \subset \boldsymbol{W}$ ). Assuming that $\boldsymbol{u} \in \boldsymbol{W}$, the first contact condition $(2.2 \mathrm{e})$ can be written as (see $[14,11])$ :

$$
\sigma^{n}(\boldsymbol{u})=\left[\sigma^{n}(\boldsymbol{u})-\gamma u^{n}\right]_{\mathbb{R}^{-}}=\left[P_{1, \gamma}^{n}(\boldsymbol{u})\right]_{\mathbb{R}^{-}} .
$$




\begin{tabular}{ll}
\hline \multicolumn{1}{c}{ Notation } & \multicolumn{1}{c}{ Definition } \\
\hline $\mathcal{F}_{h}$ & Set of faces of $\mathcal{T}_{h}$ \\
$\mathcal{F}_{h}^{b}$ & Set of boundary faces, i.e., $\left\{F \in \mathcal{F}_{h}: F \subset \partial \Omega\right\}$ \\
$\mathcal{F}_{h}^{D} \cup \mathcal{F}_{h}^{N} \cup \mathcal{F}_{h}^{C}$ & Partition of $\mathcal{F}_{h}^{b}$ induced by the boundary and contact conditions \\
$\mathcal{F}_{h}^{i}$ & Set of interior faces, i.e., $\mathcal{F}_{h} \backslash \mathcal{F}_{h}^{b}$ \\
$\mathcal{F}_{T}$ & Set of faces of the element $T \in \mathcal{T}_{h}$, i.e., $\left\{F \in \mathcal{F}_{h}: F \subset \partial T\right\}$ \\
$\mathcal{F}_{T}^{\bullet}, \bullet \in\{b, D, N, C\}$ & $\mathcal{F}_{T} \cap \mathcal{F}_{h}^{\bullet}, T \in \mathcal{T}_{h}$ \\
$\mathcal{V}_{h}$ & Set of all the vertices of $\mathcal{T}_{h}$ \\
$\mathcal{V}_{h}^{b}$ & Set of boundary vertices, i.e., $\left\{\boldsymbol{a} \in \mathcal{V}_{h}: a \in \partial \Omega\right\}$ \\
$\mathcal{V}_{h}^{i}$ & Set of interior vertices, i.e., $\mathcal{V}_{h} \backslash \mathcal{V}_{h}^{b}$ \\
$\mathcal{V}_{T}$ & Set of vertices of the element $T \in \mathcal{T}_{h}$, i.e., $\left\{\boldsymbol{a} \in \mathcal{V}_{h}: a \in \boldsymbol{a} \in \partial T\right\}$ \\
$\mathcal{V}_{F}$ & Set of vertices of the mesh face $F \in \mathcal{F}_{h}$, i.e., $\left\{\boldsymbol{a} \in \mathcal{V}_{h}: \boldsymbol{a} \in \partial F\right\}$ \\
$\omega_{\boldsymbol{a}}$ & Union of the elements sharing the vertex $\boldsymbol{a} \in \mathcal{V}_{h}$, i.e., $\bigcup_{T \in \mathcal{T}_{h}, \boldsymbol{a} \in \partial T} T$ \\
\hline
\end{tabular}

Table 1: Mesh-related notations.

Remark 2 (Case $\theta=0$ ). The linear operator $P_{\theta, \gamma}^{n}$ is well defined on $\boldsymbol{V}_{h}$ since it is a subspace of the space of broken polynomials. It can be easily extended to the space $\boldsymbol{H}_{D}^{1}(\Omega)$ in the case $\theta=0$, for which $P_{0, \gamma}^{n}(\boldsymbol{v})=-\gamma v^{n}$, as $\boldsymbol{v} \in \boldsymbol{H}^{1}(\Omega)$ guarantees $\left.\boldsymbol{v}\right|_{\Gamma_{C}} \in \boldsymbol{L}^{2}\left(\Gamma_{C}\right)$ by the trace theorem.

From now on, $\gamma_{0}>0$ will denote a fixed constant called Nitsche parameter, and we suppose that $\gamma$ is the positive piecewise constant function on $\Gamma_{C}$ which satisfies: For all $T \in \mathcal{T}_{h}$ such that $\left|\partial T \cap \Gamma_{C}\right|>0$,

$$
\left.\gamma\right|_{\partial T \cap \Gamma_{C}}=\frac{\gamma_{0}}{h_{T}} .
$$

We consider the following method à la Nitsche to approximate problem (2.2), originally introduced in [11]: Find $\boldsymbol{u}_{h} \in \boldsymbol{V}_{h}$ such that

$$
a\left(\boldsymbol{u}_{h}, \boldsymbol{v}_{h}\right)-\left(\left[P_{1, \gamma}^{n}\left(\boldsymbol{u}_{h}\right)\right]_{\mathbb{R}^{-}}, v_{h}^{n}\right)_{\Gamma_{C}}=L\left(\boldsymbol{v}_{h}\right) \quad \forall \boldsymbol{v}_{h} \in \boldsymbol{V}_{h} .
$$

For the a priori analysis of the method, we refer to [11].

\section{Basic a posteriori error estimate}

In this section we derive a basic a posteriori error estimate based on the notion of equilibrated stress reconstruction.

\subsection{Error measure}

In the framework of a posteriori error estimation, the dual norm of a residual functional can be used as a measure of the error between the exact solution $\boldsymbol{u}$ of the problem and the solution $\boldsymbol{u}_{h}$ obtained with the finite element method. Denoting by $\left(\boldsymbol{H}_{D}^{1}(\Omega)\right)^{*}$ the dual space of $\boldsymbol{H}_{D}^{1}(\Omega)$, for any $\boldsymbol{w}_{h} \in \boldsymbol{V}_{h}$ the residual $\mathcal{R}\left(\boldsymbol{w}_{h}\right) \in\left(\boldsymbol{H}_{D}^{1}(\Omega)\right)^{*}$ is defined by

$$
\left\langle\mathcal{R}\left(\boldsymbol{w}_{h}\right), \boldsymbol{v}\right\rangle:=L(\boldsymbol{v})-a\left(\boldsymbol{w}_{h}, \boldsymbol{v}\right)+\left(\left[P_{1, \gamma}^{n}\left(\boldsymbol{w}_{h}\right)\right]_{\mathbb{R}^{-}}, v^{n}\right)_{\Gamma_{C}} \quad \forall \boldsymbol{v} \in \boldsymbol{H}_{D}^{1}(\Omega),
$$


where $\langle\cdot, \cdot\rangle$ denotes the duality pairing between $\boldsymbol{H}_{D}^{1}(\Omega)$ and $\left(\boldsymbol{H}_{D}^{1}(\Omega)\right)^{*}$. We equip $\boldsymbol{H}_{D}^{1}(\Omega)$ with the following mesh-dependent norm:

$$
\|\boldsymbol{v}\|\left\|^{2}:=\right\| \boldsymbol{\nabla} \boldsymbol{v} \|^{2}+|\boldsymbol{v}|_{C, h}^{2} \quad \forall \boldsymbol{v} \in \boldsymbol{H}_{D}^{1}(\Omega),
$$

where

$$
|\boldsymbol{v}|_{C, h}^{2}:=\sum_{F \in \mathcal{F}_{h}^{C}} \frac{1}{h_{F}}\|\boldsymbol{v}\|_{F}^{2} \quad \forall \boldsymbol{v} \in \boldsymbol{H}_{D}^{1}(\Omega) .
$$

It is easy to show that $|\cdot|_{C, h}$ is subadditive and absolutely homogeneous, i.e., it is a seminorm. As a consequence, also $\|\cdot\| \|$ is subadditive and absolutely homogeneous. Moreover, if we suppose $\|\boldsymbol{v}\| \mid=0$, then both $\|\boldsymbol{\nabla} \boldsymbol{v}\|$ and $|\boldsymbol{v}|_{C, h}$ are zero, and this implies $\boldsymbol{v}=\mathbf{0}$ by the Friedrichs inequality in $\boldsymbol{H}_{D}^{1}(\Omega)$, showing that $\|\cdot\| \|$ is indeed a norm on $\boldsymbol{H}_{D}^{1}(\Omega)$.

The dual norm of the residual of a function $\boldsymbol{w}_{h} \in \boldsymbol{V}_{h}$ on the normed space $\left(\boldsymbol{H}_{D}^{1}(\Omega),\|\| \cdot \|\right)$ is given by

$$
\left\|\mathcal{R}\left(\boldsymbol{w}_{h}\right)\right\|_{*}:=\sup _{\boldsymbol{v} \in \boldsymbol{H}_{D}^{1}(\Omega),\|\boldsymbol{v}\|=1}\left\langle\mathcal{R}\left(\boldsymbol{w}_{h}\right), \boldsymbol{v}\right\rangle .
$$

In what follows, the quantity $\left\|\mathcal{R}\left(\boldsymbol{u}_{h}\right)\right\|_{*}$ will be used as a measure of the error committed approximating the exact solution $\boldsymbol{u}$ with $\boldsymbol{u}_{h}$.

\subsection{A posteriori error estimate}

We start this section by introducing the concept of equilibrated stress reconstruction and the definition of five error estimators.

Definition 3 (Equilibrated stress reconstruction). We will call equilibrated stress reconstruction any second-order tensor $\sigma_{h}$ such that:

1. $\sigma_{h} \in \mathbb{H}(\operatorname{div}, \Omega)$,

2. $\left(\operatorname{div} \sigma_{h}+\boldsymbol{f}, \boldsymbol{v}\right)_{T}=0$ for every $\boldsymbol{v} \in \mathcal{P}^{0}(T)$ and every $T \in \mathcal{T}_{h}$,

3. $\left.\left(\boldsymbol{\sigma}_{h} \boldsymbol{n}\right)\right|_{F} \in \boldsymbol{L}^{2}(F)$ for every $F \in \mathcal{F}_{h}^{N} \cup \mathcal{F}_{h}^{C}$, and $\left(\boldsymbol{\sigma}_{h} \boldsymbol{n}, \boldsymbol{v}\right)_{F}=\left(\boldsymbol{g}_{N}, \boldsymbol{v}\right)_{F}$ for every $\boldsymbol{v} \in \mathcal{P}^{0}(F)$ and every $F \in \mathcal{F}_{h}^{N}$,

4. $\sigma_{h}^{t}=\mathbf{0}$ on $\Gamma_{C}$.

Given an equilibrated stress reconstruction $\sigma_{h}$, for every element $T \in \mathcal{T}_{h}$, we define the following local error estimators:

$$
\begin{aligned}
\eta_{\mathrm{osc}, T} & :=\frac{h_{T}}{\pi}\left\|\boldsymbol{f}+\operatorname{div} \boldsymbol{\sigma}_{h}\right\|_{T}, & & \text { (oscillation) } \\
\eta_{\mathrm{str}, T} & :=\left\|\boldsymbol{\sigma}_{h}-\boldsymbol{\sigma}\left(\boldsymbol{u}_{h}\right)\right\|_{T}, & & \text { (stress) } \\
\eta_{\mathrm{Neu}, T} & :=\sum_{F \in \mathcal{F}_{T}^{N}} C_{t, T, F} h_{F}^{1 / 2}\left\|\boldsymbol{g}_{N}-\boldsymbol{\sigma}_{h} \boldsymbol{n}\right\|_{F}, & & \text { (Neumann) } \\
\eta_{\mathrm{cnt}, T} & :=\sum_{F \in \mathcal{F}_{T}^{C}} h_{F}^{1 / 2}\left\|\left[P_{1, \gamma}^{n}\left(\boldsymbol{u}_{h}\right)\right]_{\mathbb{R}^{-}}-\sigma_{h}^{n}\right\|_{F} . & & \text { (contact) }
\end{aligned}
$$

Here, $C_{t, T, F}$ is the constant of the trace inequality $\left\|v-\overline{\boldsymbol{v}}_{F}\right\|_{F} \leq C_{t, T, F} h_{F}^{1 / 2}\|\boldsymbol{\nabla} \boldsymbol{v}\|_{T}$ with $\overline{\boldsymbol{v}}_{F}:=$ $\frac{1}{|F|} \int_{F} v$ valid for every $v \in H^{1}(T)$ and $F \in \mathcal{F}_{T}$; see [32, Theorem 4.6.3] or [16, Section 1.4]. 
The estimator $\eta_{\mathrm{osc}, T}$ represents the residual of the force balance equation (2.2a) inside the element $T, \eta_{\mathrm{str}, T}$ the difference between the Cauchy stress tensor computed from the approximate solution and the equilibrated stress reconstruction, $\eta_{\mathrm{Neu}, T}$ the residual of the Neumann boundary condition (2.2d), and $\eta_{\mathrm{cnt}, T}$ the residual of the normal condition (2.2e) on the contact boundary.

Theorem 4 (A posteriori error estimate for the dual norm of the residual). Let $\boldsymbol{u}_{h}$ be the solution of (2.7), $\mathcal{R}\left(\boldsymbol{u}_{h}\right)$ the residual defined by (3.1), and $\boldsymbol{\sigma}_{h}$ an equilibrated stress reconstruction in the sense of Definition 3. Then,

$$
\left\|\mathcal{R}\left(\boldsymbol{u}_{h}\right)\right\|_{*} \leq\left(\sum_{T \in \mathcal{T}_{h}}\left(\left(\eta_{\mathrm{osc}, T}+\eta_{\mathrm{str}, T}+\eta_{\mathrm{Neu}, T}\right)^{2}+\left(\eta_{\mathrm{cnt}, T}\right)^{2}\right)\right)^{1 / 2} .
$$

Proof. Thanks to the regularity of $\sigma_{h}$ and of its normal trace (see Properties 1. and 3. in Definition 3 ), the following Green formula holds:

$$
\left(\sigma_{h}, \boldsymbol{\nabla} \boldsymbol{v}\right)=-\left(\operatorname{div} \sigma_{h}, \boldsymbol{v}\right)+\left(\sigma_{h} \boldsymbol{n}, \boldsymbol{v}\right)_{\Gamma_{N}}+\left(\sigma_{h}^{n}, v^{n}\right)_{\Gamma_{C}} \quad \forall \boldsymbol{v} \in \boldsymbol{H}_{D}^{1}(\Omega),
$$

where we have also used the decomposition (2.1) of the normal stress reconstruction $\boldsymbol{\sigma}_{h} \boldsymbol{n}$ into normal and tangential components on the contact boundary $\Gamma_{C}$, and the fact that $\left.\sigma_{h}^{t}\right|_{\Gamma_{C}}=\mathbf{0}$ thanks to Property 4. in Definition 3. Now, fix $\boldsymbol{v} \in \boldsymbol{H}_{D}^{1}(\Omega)$ such that $\|\boldsymbol{v}\|^{2}=\|\nabla \boldsymbol{v}\|^{2}+|\boldsymbol{v}|_{C, h}^{2}=1$ and consider the argument of the supremum in the definition (3.4) of the dual norm of the residual. Expanding $L(\cdot)$ and $a(\cdot, \cdot)$ according to their definition (2.4), adding and subtracting the term $\left(\sigma_{h}, \boldsymbol{\nabla} \boldsymbol{v}\right)$, using the symmetry of $\boldsymbol{\sigma}\left(\boldsymbol{u}_{h}\right)$, and applying Green's formula (3.5), we obtain

$$
\begin{aligned}
\left\langle\mathcal{R}\left(\boldsymbol{u}_{h}\right), \boldsymbol{v}\right\rangle= & (\boldsymbol{f}, \boldsymbol{v})+\left(\boldsymbol{g}_{N}, \boldsymbol{v}\right)_{\Gamma_{N}}-\left(\boldsymbol{\sigma}\left(\boldsymbol{u}_{h}\right), \boldsymbol{\varepsilon}(\boldsymbol{v})\right)+\left(\left[P_{1, \gamma}^{n}\left(\boldsymbol{u}_{h}\right)\right]_{\mathbb{R}^{-}}, v^{n}\right)_{\Gamma_{C}} \\
& +\left(\boldsymbol{\sigma}_{h}, \boldsymbol{\nabla} \boldsymbol{v}\right)-\left(\sigma_{h}, \boldsymbol{\nabla} \boldsymbol{v}\right) \\
= & \left(\boldsymbol{f}+\operatorname{div} \boldsymbol{\sigma}_{h}, \boldsymbol{v}\right)+\left(\boldsymbol{\sigma}_{h}-\boldsymbol{\sigma}\left(\boldsymbol{u}_{h}\right), \boldsymbol{\nabla} \boldsymbol{v}\right)+\left(\boldsymbol{g}_{N}-\boldsymbol{\sigma}_{h} \boldsymbol{n}, \boldsymbol{v}\right)_{\Gamma_{N}} \\
& +\left(\left[P_{1, \gamma}^{n}\left(\boldsymbol{u}_{h}\right)\right]_{\mathbb{R}^{-}}-\sigma_{h}^{n}, v^{n}\right)_{\Gamma_{C}} \\
= & : \mathfrak{I}_{1}+\cdots+\mathfrak{T}_{4} .
\end{aligned}
$$

We estimate each term separately. Denoting by $\Pi_{T}^{0}$ the $L^{2}$-orthogonal projection onto $\mathcal{P}^{0}(T)$, and using Property 2. of Definition 3 with test function $\boldsymbol{\Pi}_{T}^{0} \boldsymbol{v} \in \mathcal{P}^{0}(T)$, the Cauchy-Schwarz inequality, and the Poincaré inequality $\left\|\boldsymbol{v}-\boldsymbol{\Pi}_{T}^{0} \boldsymbol{v}\right\|_{T} \leq h_{T} \pi^{-1}\|\boldsymbol{\nabla} \boldsymbol{v}\|_{T}$, the first term becomes

$$
\begin{aligned}
\mathfrak{I}_{1} & =\sum_{T \in \mathcal{T}_{h}}\left(f+\operatorname{div} \sigma_{h}, \boldsymbol{v}-\Pi_{T}^{0} \boldsymbol{v}\right)_{T} \leq \sum_{T \in \mathcal{T}_{h}}\left\|f+\operatorname{div} \sigma_{h}\right\|_{T}\left\|\boldsymbol{v}-\Pi_{T}^{0} \boldsymbol{v}\right\|_{T} \\
& \leq \sum_{T \in \mathcal{T}_{h}} \frac{h_{T}}{\pi}\left\|f+\operatorname{div} \sigma_{h}\right\|_{T}\|\nabla \boldsymbol{v}\|_{T}=\sum_{T \in \mathcal{T}_{h}} \eta_{\mathrm{osc}, T}\|\nabla \boldsymbol{v}\|_{T} .
\end{aligned}
$$

For the second term, we simply use the Cauchy-Schwarz inequality:

$$
\mathfrak{T}_{2} \leq \sum_{T \in \mathcal{T}_{h}}\left\|\sigma_{h}-\sigma\left(\boldsymbol{u}_{h}\right)\right\|_{T}\|\nabla v\|_{T}=\sum_{T \in \mathcal{T}_{h}} \eta_{\mathrm{str}, T}\|\nabla v\|_{T}
$$

Denoting by $\boldsymbol{\Pi}_{F}^{0}$ the $L^{2}$-orthogonal projection onto $\boldsymbol{P}^{0}(F)$, and using Property 3. of Definition 3 with $\boldsymbol{\Pi}_{F}^{0} \boldsymbol{v} \in \mathcal{P}^{0}(F)$ as a test function, the Cauchy-Schwarz inequality, and the trace inequality 
$\left\|\boldsymbol{v}-\boldsymbol{\Pi}_{F}^{0} \boldsymbol{v}\right\|_{F} \leq C_{t, T, F} h_{F}^{1 / 2}\|\boldsymbol{\nabla} \boldsymbol{v}\|_{T}, F \subset \partial T$, we have

$$
\begin{aligned}
\mathfrak{I}_{3} & =\sum_{T \in \mathcal{T}_{h}} \sum_{F \in \mathcal{F}_{T}^{N}}\left(\boldsymbol{g}_{N}-\boldsymbol{\sigma}_{h} \boldsymbol{n}, \boldsymbol{v}-\boldsymbol{\Pi}_{F}^{0} \boldsymbol{v}\right)_{F} \leq \sum_{T \in \mathcal{T}_{h}} \sum_{F \in \mathcal{F}_{T}^{N}}\left\|\boldsymbol{g}_{N}-\boldsymbol{\sigma}_{h} \boldsymbol{n}\right\|_{F}\left\|\boldsymbol{v}-\boldsymbol{\Pi}_{F}^{0} \boldsymbol{v}\right\|_{F} \\
& \leq \sum_{T \in \mathcal{T}_{h}} \sum_{F \in \mathcal{F}_{T}^{N}} C_{t, T, F} h_{F}^{1 / 2}\left\|\boldsymbol{g}_{N}-\boldsymbol{\sigma}_{h} \boldsymbol{n}\right\|_{F}\|\boldsymbol{\nabla} \boldsymbol{v}\|_{T}=\sum_{T \in \mathcal{T}_{h}} \eta_{\mathrm{Neu}, T}\|\boldsymbol{\nabla} \boldsymbol{v}\|_{T}
\end{aligned}
$$

where we recall that, for any $T \in \mathcal{T}_{h}, \mathcal{F}_{T}^{N}$ collects the Neumann faces of $T$ contained in $\mathcal{F}_{h}^{N}$. Finally, we consider the term on $\Gamma_{C}$. We define, for all $T \in \mathcal{T}_{h}$, the local counterpart of the seminorm (3.3)

$$
|v|_{C, T}^{2}:=\sum_{F \in \mathcal{F}_{T}^{C}} \frac{1}{h_{F}}\|v\|_{F}^{2},
$$

where $\mathcal{F}_{T}^{C}$ is the (possibly empty) set collecting the contact faces of $T$ contained in $\partial T \cap \Gamma_{C}$. Then, using the Cauchy-Schwarz inequality, we obtain

$$
\begin{aligned}
\mathfrak{I}_{4} & \leq \sum_{T \in \mathcal{T}_{h}} \sum_{F \in \mathcal{F}_{T}^{C}}\left\|\left[P_{1, \gamma}^{n}\left(\boldsymbol{u}_{h}\right)\right]_{\mathbb{R}^{-}}-\sigma_{h}^{n}\right\|_{F}\left\|v^{n}\right\|_{F} \leq \sum_{T \in \mathcal{T}_{h}} \sum_{F \in \mathcal{F}_{T}^{C}} h_{F}^{1 / 2}\left\|\left[P_{1, \gamma}^{n}\left(\boldsymbol{u}_{h}\right)\right]_{\mathbb{R}^{-}}-\sigma_{h}^{n}\right\|_{F}|\boldsymbol{v}|_{C, T} \\
& =\sum_{T \in \mathcal{T}_{h}} \eta_{\mathrm{cnt}, T}|\boldsymbol{v}|_{C, T} .
\end{aligned}
$$

Let, for the sake of brevity, $\eta_{a, T}:=\eta_{\mathrm{osc}, T}+\eta_{\mathrm{str}, T}+\eta_{\mathrm{Neu}, T}$ for any element $T \in \mathcal{T}_{h}$. Combining the above results and applying the Cauchy-Schwarz inequality, we obtain

$$
\begin{aligned}
\left\|\mathcal{R}\left(\boldsymbol{u}_{h}\right)\right\|_{*} & \leq \sup _{\boldsymbol{v} \in \boldsymbol{H}_{D}^{1}(\Omega),\|\boldsymbol{v}\| \|=1}\left\{\sum_{T \in \mathcal{T}_{h}}\left(\eta_{a, T}\|\boldsymbol{\nabla} \boldsymbol{v}\|_{T}+\eta_{\mathrm{cnt}, T}|\boldsymbol{v}|_{C, T}\right)\right\} \\
& \leq \sup _{\boldsymbol{v} \in \boldsymbol{H}_{D}^{1}(\Omega),\|\boldsymbol{v}\| \|=1}\left\{\left(\sum_{T \in \mathcal{T}_{h}}\left(\left(\eta_{a, T}\right)^{2}+\left(\eta_{\mathrm{cnt}, \mathrm{T}}\right)^{2}\right)\right)^{1 / 2}\left(\sum_{T \in \mathcal{T}_{h}}\left(\|\boldsymbol{\nabla} \boldsymbol{v}\|_{T}^{2}+|\boldsymbol{v}|_{C, T}^{2}\right)\right)^{1 / 2}\right\} \\
& =\left(\sum_{T \in \mathcal{T}_{h}}\left(\left(\eta_{\mathrm{osc}, T}+\eta_{\mathrm{str}, T}+\eta_{\mathrm{Neu}, T}\right)^{2}+\left(\eta_{\mathrm{cnt}, T}\right)^{2}\right)\right)^{1 / 2} \cdot
\end{aligned}
$$

Remark 5 (A posteriori error estimate for stress reconstructions with contact friction). Even without Property 4. in Definition 3 one can easily obtain an a posteriori error estimate similarly to Theorem 4: introducing a fifth local estimator

$$
\eta_{\text {fric }, T}:=\sum_{F \in \mathcal{F}_{T}^{C}} h_{F}^{1 / 2}\left\|\sigma_{h}^{t}\right\|_{F}, \quad \text { (friction) }
$$

which represents the residual of the tangential condition (2.2f) on the contact boundary, one gets

$$
\left\|\mathcal{R}\left(\boldsymbol{u}_{h}\right)\right\|_{*} \leq\left(\sum_{T \in \mathcal{T}_{h}}\left(\left(\eta_{o s c, T}+\eta_{s t r, T}+\eta_{\text {Neu, }, T}\right)^{2}+\left(\eta_{c n t, T}+\eta_{\text {fric }, T}\right)^{2}\right)\right)^{1 / 2} .
$$

\subsection{Comparison between the residual dual norm and the energy norm}

The goal of this section is to compare the dual norm $\left\|\mathcal{R}\left(\boldsymbol{u}_{h}\right)\right\|_{*}$ with the energy norm $\left\|\boldsymbol{u}-\boldsymbol{u}_{h}\right\|_{\text {en }}$ of the error, where

$$
\|\boldsymbol{v}\|_{\mathrm{en}}^{2}:=a(\boldsymbol{v}, \boldsymbol{v})=(\boldsymbol{\sigma}(\boldsymbol{v}), \boldsymbol{\varepsilon}(\boldsymbol{v})) \quad \forall \boldsymbol{v} \in \boldsymbol{H}_{D}^{1}(\Omega) .
$$


Remark 6(Coercivity of the bilinear form $a$ ). The bilinear form $a(\cdot, \cdot)$ on the space $\left(\boldsymbol{H}_{D}^{1}(\Omega),\|\cdot\|_{1, \Omega}\right)$ is elliptic with a constant $\alpha$ which depends on the Lamé parameter $\mu$ and on the Korn constant $C_{K}$ :

$$
\alpha\|\boldsymbol{v}\|_{1, \Omega}^{2} \leq a(\boldsymbol{v}, \boldsymbol{v})=\|\boldsymbol{v}\|_{\mathrm{en}}^{2} \quad \forall \boldsymbol{v} \in \boldsymbol{H}_{D}^{1}(\Omega) .
$$

Throughout the rest of this section, we adopt the following shorthand notation: For every $a, b \in \mathbb{R}$, we write $a \lesssim b$ for $a \leq C b$ with $C>0$ independent of the mesh size $h$ and of the Nitsche parameter $\gamma_{0}$.

Theorem 7 (Control of the energy norm). Assume that the solution $\boldsymbol{u}$ of the continuous problem (2.2) belongs to $\boldsymbol{H}^{\frac{3}{2}+v}(\Omega)$ for some $v>0$, and let $\boldsymbol{u}_{h} \in \boldsymbol{V}_{h}$ be the solution of the discrete problem (2.7). Then,

$$
\alpha^{1 / 2}\left\|\boldsymbol{u}-\boldsymbol{u}_{h}\right\|_{\mathrm{en}} \lesssim\left\|\mathcal{R}\left(\boldsymbol{u}_{h}\right)\right\|_{*}+\sum_{F \in \mathcal{F}_{h}^{C}} \frac{1}{h_{F}^{1 / 2}}\left\|\sigma^{n}(\boldsymbol{u})-\left[P_{1, \gamma}^{n}\left(\boldsymbol{u}_{h}\right)\right]_{\mathbb{R}^{-}}\right\|_{F} .
$$

Furthermore, if the saturation assumption (see [9, 10])

$$
\left\|\left(\frac{\gamma_{0}}{\gamma}\right)^{1 / 2} \sigma^{n}\left(\boldsymbol{u}-\boldsymbol{u}_{h}\right)\right\|_{\Gamma_{C}} \lesssim\left\|\boldsymbol{u}-\boldsymbol{u}_{h}\right\|_{1, \Omega}
$$

holds and $\gamma_{0}$ is sufficiently large, then

$$
\left\|\boldsymbol{u}-\boldsymbol{u}_{h}\right\|_{\mathrm{en}} \lesssim\left\|\mathcal{R}\left(\boldsymbol{u}_{h}\right)\right\|_{*} .
$$

Remark 8 (Role of the regularity assumption). In Theorem 7, the solution of the contact problem (2.2) $\boldsymbol{u}$ is supposed to be sufficiently regular in order to ensure that the normal component of the Cauchy stress tensor is square-integrable on the contact boundary $\Gamma_{C}$. As a matter of fact, this regularity assumption implies $\boldsymbol{\sigma}(\boldsymbol{u}) \boldsymbol{n} \in \boldsymbol{H}^{v}\left(\Gamma_{C}\right) \subset \boldsymbol{L}^{2}\left(\Gamma_{C}\right)$.

Proof of Theorem 7. The proof adapts the ideas of [10, Theorem 3.5].

1) Proof of (3.8). Let $\boldsymbol{v}_{h} \in \boldsymbol{V}_{h}$. Using the definition (3.6) of the energy norm and the bilinearity of $a(\cdot, \cdot)$, we can write

$$
\left\|\boldsymbol{u}-\boldsymbol{u}_{h}\right\|_{\mathrm{en}}^{2}=a\left(\boldsymbol{u}-\boldsymbol{u}_{h}, \boldsymbol{u}-\boldsymbol{u}_{h}\right)=a\left(\boldsymbol{u}, \boldsymbol{u}-\boldsymbol{u}_{h}\right)-a\left(\boldsymbol{u}_{h}, \boldsymbol{u}-\boldsymbol{v}_{h}\right)-a\left(\boldsymbol{u}_{h}, \boldsymbol{v}_{h}-\boldsymbol{u}_{h}\right) .
$$

For the term $a\left(\boldsymbol{u}, \boldsymbol{u}-\boldsymbol{u}_{h}\right)$, we first use the definition (2.4) of the bilinear form $a(\cdot, \cdot)$ followed by the symmetry of the Cauchy stress tensor $\boldsymbol{\sigma}(\boldsymbol{u})$ to replace $\boldsymbol{\varepsilon}\left(\boldsymbol{u}-\boldsymbol{u}_{h}\right)$ with $\boldsymbol{\nabla}\left(\boldsymbol{u}-\boldsymbol{u}_{h}\right)$, then an integration by parts, and, finally, the fact that $\boldsymbol{u}$ satisfies (2.2) a.e. to infer:

$$
\begin{aligned}
a\left(\boldsymbol{u}, \boldsymbol{u}-\boldsymbol{u}_{h}\right) & =\left(\boldsymbol{\sigma}(\boldsymbol{u}), \boldsymbol{\varepsilon}\left(\boldsymbol{u}-\boldsymbol{u}_{h}\right)\right)=\left(\boldsymbol{\sigma}(\boldsymbol{u}), \boldsymbol{\nabla}\left(\boldsymbol{u}-\boldsymbol{u}_{h}\right)\right) \\
& =-\left(\operatorname{div} \boldsymbol{\sigma}(\boldsymbol{u}), \boldsymbol{u}-\boldsymbol{u}_{h}\right)+\left(\boldsymbol{\sigma}(\boldsymbol{u}) \boldsymbol{n}, \boldsymbol{u}-\boldsymbol{u}_{h}\right)_{\partial \Omega} \\
& =\left(\boldsymbol{f}, \boldsymbol{u}-\boldsymbol{u}_{h}\right)+\left(\boldsymbol{g}_{N}, \boldsymbol{u}-\boldsymbol{u}_{h}\right)_{\Gamma_{N}}+\left(\sigma^{n}(\boldsymbol{u}), u^{n}-u_{h}^{n}\right)_{\Gamma_{C}} .
\end{aligned}
$$

Notice that, in the last term, only the normal component of the traction appears as $\boldsymbol{\sigma}^{\boldsymbol{t}}(\boldsymbol{u})=\mathbf{0}$ on $\Gamma_{C}$ by (2.2f).

Concerning the term $a\left(\boldsymbol{u}_{h}, \boldsymbol{v}_{h}-\boldsymbol{u}_{h}\right)$ in (3.11), since $\boldsymbol{u}_{h}$ solves (2.7) and $\boldsymbol{v}_{h}-\boldsymbol{u}_{h} \in \boldsymbol{V}_{h}$, we have

$$
a\left(\boldsymbol{u}_{h}, \boldsymbol{v}_{h}-\boldsymbol{u}_{h}\right)=\left(\boldsymbol{f}, \boldsymbol{v}_{h}-\boldsymbol{u}_{h}\right)+\left(\boldsymbol{g}_{N}, \boldsymbol{v}_{h}-\boldsymbol{u}_{h}\right)_{\Gamma_{N}}+\left(\left[P_{1, \gamma}^{n}\left(\boldsymbol{u}_{h}\right)\right]_{\mathbb{R}^{-}}, v_{h}^{n}-u_{h}^{n}\right)_{\Gamma_{C}},
$$


where we have additionally expanded the linear form $L(\cdot)$ according to its definition (2.4).

Plugging (3.12) and (3.13) into (3.11), we then obtain

$$
\begin{aligned}
\left\|\boldsymbol{u}-\boldsymbol{u}_{h}\right\|_{\mathrm{en}}^{2}= & \left(\boldsymbol{f}, \boldsymbol{u}-\boldsymbol{v}_{h}\right)+\left(\boldsymbol{g}_{N}, \boldsymbol{u}-\boldsymbol{v}_{h}\right)_{\Gamma_{N}}+\left(\sigma^{n}(\boldsymbol{u}), u^{n}-u_{h}^{n}\right)_{\Gamma_{C}} \\
& -\left(\boldsymbol{\sigma}\left(\boldsymbol{u}_{h}\right), \boldsymbol{\varepsilon}\left(\boldsymbol{u}-\boldsymbol{v}_{h}\right)\right)-\left(\left[P_{1, \gamma}^{n}\left(\boldsymbol{u}_{h}\right)\right]_{\mathbb{R}^{-}}, v_{h}^{n}-u_{h}^{n}\right)_{\Gamma_{C}}=\mathfrak{I}_{1}+\mathfrak{T}_{2}
\end{aligned}
$$

where, recalling the definition (3.1) of the residual,

$$
\mathfrak{I}_{1}:=\left\langle\mathcal{R}\left(\boldsymbol{u}_{h}\right), \boldsymbol{u}-\boldsymbol{v}_{h}\right\rangle, \quad \mathfrak{I}_{2}:=\left(\sigma^{n}(\boldsymbol{u})-\left[P_{1, \gamma}^{n}\left(\boldsymbol{u}_{h}\right)\right]_{\mathbb{R}^{-}}, u^{n}-u_{h}^{n}\right)_{\Gamma_{C}} .
$$

Notice that the reformulation of $\mathfrak{I}_{1}$ in terms of the residual $\mathcal{R}\left(\boldsymbol{u}_{h}\right)$ is a consequence of (3.1) and of the definition (2.4) of the linear form $L(\cdot)$ and of the bilinear form $a(\cdot, \cdot)$.

For the first term, we can write, by definition (3.4) of the dual norm,

$$
\mathfrak{I}_{1} \leq\left\|\boldsymbol{u}-\boldsymbol{v}_{h}\right\|\|\| \mathcal{R}\left(\boldsymbol{u}_{h}\right) \|_{*} .
$$

We now want to show that $\left\|\boldsymbol{u}-\boldsymbol{v}_{h}\right\| \mid \lesssim\left\|\boldsymbol{u}-\boldsymbol{u}_{h}\right\|_{\text {en }}$ for a properly selected function $\boldsymbol{v}_{h}$. From now on, we fix $\boldsymbol{v}_{h}=\boldsymbol{u}_{h}+\mathcal{I}_{h}\left(\boldsymbol{u}-\boldsymbol{u}_{h}\right)$, where $\mathcal{I}_{h}: \boldsymbol{H}_{D}^{1}(\Omega) \rightarrow \boldsymbol{V}_{h}$ is the quasi-interpolation operator defined in [3, Eq. (4.11)], whose main properties are summarized in [10, Lemma 2.1]. We analyze separately the two parts composing the norm $\|\cdot\| \| \mid\left(\right.$ see (3.2)). For the $\boldsymbol{H}^{1}$-seminorm, we use, in this order, the triangle inequality, the choice of $\boldsymbol{v}_{h}$, the boundedness in the $\boldsymbol{H}^{1}$-norm of the operator $\mathcal{I}_{h}$ (i.e., $\left\|\mathcal{I}_{h} \boldsymbol{v}\right\|_{1, \Omega} \lesssim\|\boldsymbol{v}\|_{1, \Omega}$ for every $\boldsymbol{v} \in \boldsymbol{H}_{D}^{1}(\Omega)$ ), and the ellipticity (3.7) of the bilinear form $a(\cdot, \cdot)$ to write:

$$
\begin{aligned}
\left\|\boldsymbol{\nabla}\left(\boldsymbol{u}-\boldsymbol{v}_{h}\right)\right\| & \leq\left\|\boldsymbol{u}-\boldsymbol{v}_{h}\right\|_{1, \Omega} \leq\left\|\boldsymbol{u}-\boldsymbol{u}_{h}\right\|_{1, \Omega}+\left\|\boldsymbol{u}_{h}-\boldsymbol{v}_{h}\right\|_{1, \Omega} \\
& =\left\|\boldsymbol{u}-\boldsymbol{u}_{h}\right\|_{1, \Omega}+\left\|\mathcal{I}_{h}\left(\boldsymbol{u}-\boldsymbol{u}_{\boldsymbol{h}}\right)\right\|_{1, \Omega} \lesssim\left\|\boldsymbol{u}-\boldsymbol{u}_{h}\right\|_{1, \Omega} \leq \alpha^{-1 / 2}\left\|\boldsymbol{u}-\boldsymbol{u}_{h}\right\|_{\mathrm{en}} .
\end{aligned}
$$

Next, using the definition (3.3) of the seminorm $|\cdot|_{C, h}$, the choice of $\boldsymbol{v}_{h}$, and the ellipticity (3.7) of $a(\cdot, \cdot)$, we obtain:

$$
\begin{aligned}
\left|\boldsymbol{u}-\boldsymbol{v}_{h}\right|_{C, h}^{2} & =\sum_{F \in \mathcal{F}_{h}^{C}} \frac{1}{h_{F}}\left\|\boldsymbol{u}-\boldsymbol{v}_{h}\right\|_{F}^{2}=\sum_{F \in \mathcal{F}_{h}^{C}} \frac{1}{h_{F}}\left\|\boldsymbol{u}-\boldsymbol{u}_{h}-\mathcal{I}_{h}\left(\boldsymbol{u}-\boldsymbol{u}_{h}\right)\right\|_{F}^{2} \\
& \lesssim \sum_{F \in \mathcal{F}_{h}^{C}}\left\|\boldsymbol{u}-\boldsymbol{u}_{h}\right\|_{1, \tilde{\omega}_{F}}^{2} \lesssim\left\|\boldsymbol{u}-\boldsymbol{u}_{h}\right\|_{1, \Omega}^{2} \leq \alpha^{-1}\left\|\boldsymbol{u}-\boldsymbol{u}_{h}\right\|_{\mathrm{en}}^{2},
\end{aligned}
$$

where, to pass to the second line, we have used the following trace approximation property of $\mathcal{I}_{h}$ (see [10, Lemma 2.1]): For all $F \in \mathcal{F}_{h}$,

$$
\left\|\boldsymbol{v}-\mathcal{I}_{h} \boldsymbol{v}\right\|_{F} \lesssim h_{F}^{1 / 2}\|\boldsymbol{v}\|_{1, \tilde{\omega}_{F}} \quad \forall \boldsymbol{v} \in \boldsymbol{H}_{D}^{1}(\Omega),
$$

with $\tilde{\omega}_{F}$ standing for the union of the mesh elements sharing at least one vertex with $F$, see Figure 1. Recalling the definition (3.2) of the triple norm, squaring (3.16) and summing it to (3.17), and taking the square root of the resulting inequality, we conclude that

$$
\left\|\boldsymbol{u}-\boldsymbol{v}_{h}\right\|\left\|=\left(\left\|\boldsymbol{\nabla}\left(\boldsymbol{u}-\boldsymbol{v}_{h}\right)\right\|^{2}+\left|\boldsymbol{u}-\boldsymbol{v}_{h}\right|_{C, h}^{2}\right)^{1 / 2} \lesssim \alpha^{-1 / 2}\right\| \boldsymbol{u}-\boldsymbol{u}_{h} \|_{\mathrm{en}} .
$$

Combining this bound with (3.15), we obtain

$$
\mathfrak{I}_{1} \lesssim \alpha^{-1 / 2}\left\|\boldsymbol{u}-\boldsymbol{u}_{h}\right\|_{\text {en }}\left\|\mathcal{R}\left(\boldsymbol{u}_{h}\right)\right\|_{*} .
$$



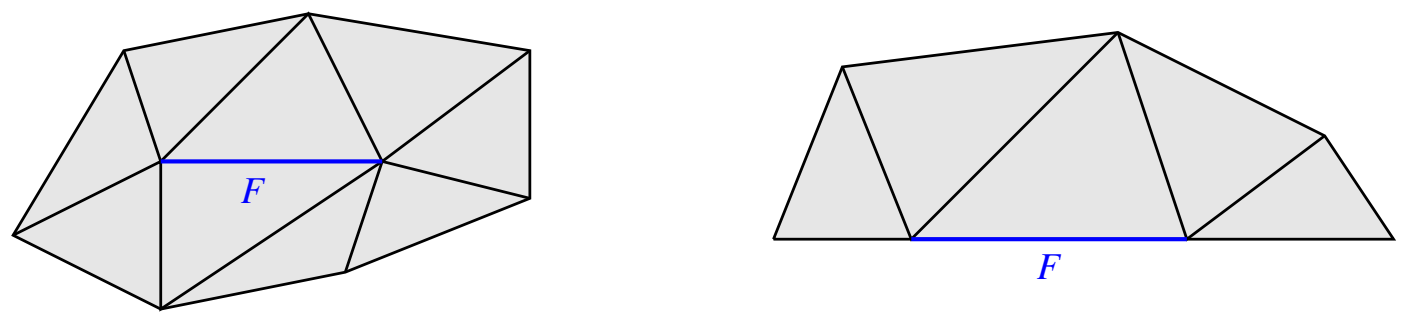

Figure 1: Illustration of $\tilde{\omega}_{F}$ for $F \in \mathcal{F}_{h}^{i}(l e f t)$ and for $F \in \mathcal{F}_{h}^{b}($ right $)$.

We now consider the term $\mathfrak{I}_{2}$. Using the Cauchy-Schwarz and trace inequalities, we have

$$
\begin{aligned}
\mathfrak{I}_{2} & =\sum_{F \in \mathcal{F}_{h}^{C}}\left(\sigma^{n}(\boldsymbol{u})-\left[P_{1, \gamma}^{n}\left(\boldsymbol{u}_{h}\right)\right]_{\mathbb{R}^{-}}, u^{n}-u_{h}^{n}\right)_{F} \\
& \lesssim\left(\sum_{F \in \mathscr{F}_{h}^{C}} \frac{1}{h_{F}}\left\|\sigma^{n}(\boldsymbol{u})-\left[P_{1, \gamma}^{n}\left(\boldsymbol{u}_{h}\right)\right]_{\mathbb{R}^{-}}\right\|_{F}^{2}\right)^{1 / 2}\left\|\boldsymbol{u}-\boldsymbol{u}_{h}\right\|_{1, \Omega} \\
& \lesssim \alpha^{-1 / 2}\left(\sum_{F \in \mathcal{F}_{h}^{C}} \frac{1}{h_{F}}\left\|\sigma^{n}(\boldsymbol{u})-\left[P_{1, \gamma}^{n}\left(\boldsymbol{u}_{h}\right)\right]_{\mathbb{R}^{-}}\right\|_{F}^{2}\right)^{1 / 2}\left\|\boldsymbol{u}-\boldsymbol{u}_{h}\right\|_{\mathrm{en}} .
\end{aligned}
$$

Inserting (3.18) and (3.19) into (3.14), we obtain (3.8).

2) Proof of (3.10). For the second part of the theorem, we work under the saturation assumption (3.9). Using the contact condition $\sigma^{n}(\boldsymbol{u})=\left[P_{1, \gamma}^{n}(\boldsymbol{u})\right]_{\mathbb{R}^{-}}$(see (2.6)), and the definition (2.5) of the operator $P_{1, \gamma}^{n}$, we have

$$
\begin{aligned}
\mathfrak{T}_{2}= & \left(\left[P_{1, \gamma}^{n}(\boldsymbol{u})\right]_{\mathbb{R}^{-}}-\left[P_{1, \gamma}^{n}\left(\boldsymbol{u}_{h}\right)\right]_{\mathbb{R}^{-}}, u^{n}-u_{h}^{n}\right)_{\Gamma_{C}} \\
= & \left(\left[P_{1, \gamma}^{n}(\boldsymbol{u})\right]_{\mathbb{R}^{-}}-\left[P_{1, \gamma}^{n}\left(\boldsymbol{u}_{h}\right)\right]_{\mathbb{R}^{-}}, \frac{1}{\gamma}\left[\gamma\left(u^{n}-u_{h}^{n}\right)-\sigma^{n}\left(\boldsymbol{u}-\boldsymbol{u}_{h}\right)+\sigma^{n}\left(\boldsymbol{u}-\boldsymbol{u}_{h}\right)\right]\right)_{\Gamma_{C}} \\
= & -\left(\left[P_{1, \gamma}^{n}(\boldsymbol{u})\right]_{\mathbb{R}^{-}}-\left[P_{1, \gamma}^{n}\left(\boldsymbol{u}_{h}\right)\right]_{\mathbb{R}^{-}}, \frac{1}{\gamma}\left(P_{1, \gamma}^{n}(\boldsymbol{u})-P_{1, \gamma}\left(\boldsymbol{u}_{h}\right)\right)\right)_{\Gamma_{C}} \\
& +\left(\left[P_{1, \gamma}^{n}(\boldsymbol{u})\right]_{\mathbb{R}^{-}}-\left[P_{1, \gamma}^{n}\left(\boldsymbol{u}_{h}\right)\right]_{\mathbb{R}^{-}}, \frac{1}{\gamma} \sigma^{n}\left(\boldsymbol{u}-\boldsymbol{u}_{h}\right)\right)_{\Gamma_{C}} .
\end{aligned}
$$

Due to the fact that $a[a]_{\mathbb{R}^{-}}=\left([a]_{\mathbb{R}^{-}}\right)^{2}$ and $a[b]_{\mathbb{R}^{-}} \leq[a]_{\mathbb{R}^{-}}[b]_{\mathbb{R}^{-}}$, it follows that

$$
(a-b)\left([a]_{\mathbb{R}^{-}}-[b]_{\mathbb{R}^{-}}\right)=a[a]_{\mathbb{R}^{-}}+b[b]_{\mathbb{R}^{-}}-a[b]_{\mathbb{R}^{-}}-b[a]_{\mathbb{R}^{-}} \geq\left([a]_{\mathbb{R}^{-}}-[b]_{\mathbb{R}^{-}}\right)^{2}
$$

for every $a, b \in \mathbb{R}$. Using the latter inequality with $(a, b)=\left(P_{1, \gamma}^{n}(\boldsymbol{u}), P_{1, \gamma}^{n}\left(\boldsymbol{u}_{h}\right)\right)$ for the first term in (3.20) and the Cauchy-Schwarz inequality for the second one, we have

$$
\begin{aligned}
\mathfrak{I}_{2} \leq & -\left\|\gamma^{-1 / 2}\left(\left[P_{1, \gamma}^{n}(\boldsymbol{u})\right]_{\mathbb{R}^{-}}-\left[P_{1, \gamma}^{n}\left(\boldsymbol{u}_{h}\right)\right]_{\mathbb{R}^{-}}\right)\right\|_{\Gamma_{C}}^{2} \\
& +\left\|\gamma^{-1 / 2}\left(\left[P_{1, \gamma}^{n}(\boldsymbol{u})\right]_{\mathbb{R}^{-}}-\left[P_{1, \gamma}^{n}\left(\boldsymbol{u}_{h}\right)\right]_{\mathbb{R}^{-}}\right)\right\|_{\Gamma_{C}}\left\|\gamma^{-1 / 2} \sigma^{n}\left(\boldsymbol{u}-\boldsymbol{u}_{h}\right)\right\|_{\Gamma_{C}} .
\end{aligned}
$$

We continue using the generalized Young inequality $a b \leq a^{2}+b^{2} / 4$ for the second term followed by the saturation assumption (3.9) to write:

$$
\mathfrak{I}_{2} \leq \frac{1}{4 \gamma_{0}}\left\|\left(\frac{\gamma_{0}}{\gamma}\right)^{1 / 2} \sigma^{n}\left(\boldsymbol{u}-\boldsymbol{u}_{h}\right)\right\|_{\Gamma_{C}}^{2} \lesssim \frac{1}{4 \gamma_{0}}\left\|\boldsymbol{u}-\boldsymbol{u}_{h}\right\|_{1, \Omega}^{2} \leq \frac{1}{4 \gamma_{0} \alpha}\left\|\boldsymbol{u}-\boldsymbol{u}_{h}\right\|_{\mathrm{en}}^{2} .
$$


Combining (3.14), (3.18), and (3.21) we finally get, for a suitable real number $C>0$,

$$
\left(\alpha^{1 / 2}-\frac{C}{4 \gamma_{0} \alpha}\right)\left\|\boldsymbol{u}-\boldsymbol{u}_{h}\right\|_{\mathrm{en}}^{2} \leq C\left\|\boldsymbol{u}-\boldsymbol{u}_{h}\right\|_{\mathrm{en}}\left\|\mathcal{R}\left(\boldsymbol{u}_{h}\right)\right\|_{*}
$$

and, taking $\gamma_{0}$ sufficiently large,

$$
\left\|\boldsymbol{u}-\boldsymbol{u}_{h}\right\|_{\mathrm{en}} \lesssim\left\|\mathcal{R}\left(\boldsymbol{u}_{h}\right)\right\|_{*},
$$

thus concluding the proof of (3.10).

Theorem 9 (Control of the dual norm of the residual). Assume that the solution $\boldsymbol{u}$ of the continuous problem (2.2) belongs to $\boldsymbol{H}^{\frac{3}{2}+v}(\Omega)$ for some $v>0$, and let $\boldsymbol{u}_{h} \in \boldsymbol{V}_{h}$ be the solution of the discrete problem (2.7). Then,

$$
\left\|\mathcal{R}\left(\boldsymbol{u}_{h}\right)\right\|_{*} \leq(d \lambda+4 \mu)^{1 / 2}\left\|\boldsymbol{u}-\boldsymbol{u}_{h}\right\|_{\text {en }}+\left(\sum_{F \in \mathcal{F}_{h}^{C}} h_{F}\left\|\sigma^{n}(\boldsymbol{u})-\left[P_{1, \gamma}^{n}\left(\boldsymbol{u}_{h}\right)\right]_{\mathbb{R}^{-}}\right\|_{F}^{2}\right)^{1 / 2} .
$$

Moreover, if the saturation assumption (3.9) holds, then

$$
\left\|\mathcal{R}\left(\boldsymbol{u}_{h}\right)\right\|_{*} \lesssim\left[(d \lambda+4 \mu)^{1 / 2}+\alpha^{-1 / 2}\right]\left\|\boldsymbol{u}-\boldsymbol{u}_{h}\right\|_{\text {en }}+\gamma_{0}\left(\sum_{F \in \mathcal{F}_{h}^{C}} \frac{1}{h_{F}}\left\|\boldsymbol{u}-\boldsymbol{u}_{h}\right\|_{F}^{2}\right)^{1 / 2} .
$$

Proof. 1) Proof of (3.22). By definition (3.1) of the residual together with (2.2) (valid almost everywhere), and Green's formula, it holds: For any $\boldsymbol{v} \in \boldsymbol{H}_{D}^{1}(\Omega)$,

$$
\begin{aligned}
\left\langle\mathcal{R}\left(\boldsymbol{u}_{h}\right), \boldsymbol{v}\right\rangle & =(\boldsymbol{f}, \boldsymbol{v})+\left(\boldsymbol{g}_{N}, \boldsymbol{v}\right)_{\Gamma_{N}}-\left(\boldsymbol{\sigma}\left(\boldsymbol{u}_{h}\right), \boldsymbol{\varepsilon}(\boldsymbol{v})\right)+\left(\left[P_{1, \gamma}^{n}\left(\boldsymbol{u}_{h}\right)\right]_{\mathbb{R}^{-}}, v^{n}\right)_{\Gamma_{C}} \\
& =\left(\boldsymbol{\sigma}\left(\boldsymbol{u}-\boldsymbol{u}_{h}\right), \boldsymbol{\varepsilon}(\boldsymbol{v})\right)-\left(\sigma^{n}(\boldsymbol{u})-\left[P_{1, \gamma}^{n}\left(\boldsymbol{u}_{h}\right)\right]_{\mathbb{R}^{-}}, v^{n}\right)_{\Gamma_{C}} .
\end{aligned}
$$

Then, using the symmetry of the Cauchy stress tensor $\boldsymbol{\sigma}\left(\boldsymbol{u}-\boldsymbol{u}_{h}\right)$, the Cauchy-Schwarz inequality, and the definition (3.2) of the norm $\|\boldsymbol{v}\|$, and additionally observing that

$$
\left\|\boldsymbol{\sigma}\left(\boldsymbol{u}-\boldsymbol{u}_{h}\right)\right\| \leq(d \lambda+4 \mu)^{1 / 2}\left\|\boldsymbol{u}-\boldsymbol{u}_{h}\right\|_{\mathrm{en}},
$$

we have

$$
\begin{aligned}
\left\langle\mathcal{R}\left(\boldsymbol{u}_{h}\right), \boldsymbol{v}\right\rangle & \leq\left\|\boldsymbol{\sigma}\left(\boldsymbol{u}-\boldsymbol{u}_{h}\right)\right\|\|\boldsymbol{\nabla} \boldsymbol{v}\|+\sum_{F \in \mathcal{F}_{h}^{C}}\left\|\sigma^{n}(\boldsymbol{u})-\left[P_{1, \gamma}^{n}\left(\boldsymbol{u}_{h}\right)\right]_{\mathbb{R}^{-}}\right\|_{F}\left\|v^{n}\right\|_{F} \\
& \leq(d \lambda+4 \mu)^{1 / 2}\left\|\boldsymbol{u}-\boldsymbol{u}_{h}\right\|_{\mathrm{en}}\|\boldsymbol{\nabla} \boldsymbol{v}\|+\sum_{F \in \mathcal{F}_{h}^{C}} h_{F}^{1 / 2}\left\|\sigma^{n}(\boldsymbol{u})-\left[P_{1, \gamma}^{n}\left(\boldsymbol{u}_{h}\right)\right]_{\mathbb{R}^{-}}\right\|_{F} \frac{1}{h_{F}^{1 / 2}}\|\boldsymbol{v}\|_{F} \\
& \leq\left[(d \lambda+4 \mu)^{1 / 2}\left\|\boldsymbol{u}-\boldsymbol{u}_{h}\right\|_{\mathrm{en}}+\left(\sum_{F \in \mathcal{F}_{h}^{C}} h_{F}\left\|\sigma^{n}(\boldsymbol{u})-\left[P_{1, \gamma}^{n}\left(\boldsymbol{u}_{h}\right)\right]_{\mathbb{R}^{-}}\right\|_{F}^{2}\right)^{1 / 2}\right]\|\boldsymbol{v}\| .
\end{aligned}
$$

By definition (3.4) of the dual norm, this yields (3.22). 
2) Proof of (3.23). Under the saturation assumption (3.9), starting from (3.22) and using (2.6), we obtain, for all $F \in \mathcal{F}_{h}^{C}$,

$$
\begin{aligned}
h_{F}\left\|\sigma^{n}(\boldsymbol{u})-\left[P_{1, \gamma}^{n}\left(\boldsymbol{u}_{h}\right)\right]_{\mathbb{R}^{-}}\right\|_{F}^{2}=h_{F}\left\|\left[P_{1, \gamma}^{n}(\boldsymbol{u})\right]_{\mathbb{R}^{-}}-\left[P_{1, \gamma}^{n}\left(\boldsymbol{u}_{h}\right)\right]_{\mathbb{R}^{-}}\right\|_{F}^{2} \\
\leq h_{F}\left\|P_{1, \gamma}^{n}(\boldsymbol{u})-P_{1, \gamma}^{n}\left(\boldsymbol{u}_{h}\right)\right\|_{F}^{2} \lesssim h_{F}\left\|\boldsymbol{\sigma}\left(\boldsymbol{u}-\boldsymbol{u}_{h}\right)\right\|_{F}^{2}+h_{F}\left\|\gamma\left(\boldsymbol{u}-\boldsymbol{u}_{h}\right)\right\|_{F}^{2},
\end{aligned}
$$

where we have applied the property $\left([a]_{\mathbb{R}^{-}}-[b]_{\mathbb{R}^{-}}\right)^{2} \leq(a-b)^{2}$ valid for any $a, b \in \mathbb{R}$, with $(a, b)=\left(P_{1, \gamma}^{n}(\boldsymbol{u}), P_{1, \gamma}^{n}\left(\boldsymbol{u}_{h}\right)\right)$ to pass to the second line and the triangle inequality to conclude. Then, using the saturation assumption (3.9) together with the ellipticity property (3.7) and the choice of $\gamma$, we obtain:

$$
\begin{aligned}
& \left(\sum_{F \in \mathcal{F}_{h}^{C}} h_{F}\left\|\sigma^{n}(\boldsymbol{u})-\left[P_{1, \gamma}^{n}\left(\boldsymbol{u}_{h}\right)\right]_{\mathbb{R}^{-}}\right\|_{F}^{2}\right)^{1 / 2} \\
& \quad \lesssim\left(\sum_{F \in \mathcal{F}_{h}^{C}} h_{F}\left\|\boldsymbol{\sigma}\left(\boldsymbol{u}-\boldsymbol{u}_{h}\right)\right\|_{F}^{2}+\sum_{F \in \mathcal{F}_{h}^{C}} h_{F}\left\|\gamma\left(\boldsymbol{u}-\boldsymbol{u}_{h}\right)\right\|_{F}^{2}\right)^{1 / 2} \\
& \quad \leq\left(\sum_{T \in \mathcal{T}_{h}} \sum_{F \in \mathcal{F}_{T}^{C}} h_{T}\left\|\boldsymbol{\sigma}\left(\boldsymbol{u}-\boldsymbol{u}_{h}\right)\right\|_{F}^{2}\right)^{1 / 2}+\left(\sum_{T \in \mathcal{T}_{h}} \sum_{F \in \mathcal{F}_{T}^{C}} h_{F}\left(\frac{\gamma_{0}}{h_{T}}\right)^{2}\left\|\boldsymbol{u}-\boldsymbol{u}_{h}\right\|_{F}^{2}\right)^{1 / 2} \\
& \quad \leq\left\|\left(\frac{\gamma_{0}}{\gamma}\right)^{1 / 2} \boldsymbol{\sigma}\left(\boldsymbol{u}-\boldsymbol{u}_{h}\right)\right\|_{\Gamma_{C}}+\gamma_{0}\left(\sum_{F \in \mathcal{F}_{h}^{C}} \frac{1}{h_{F}}\left\|\boldsymbol{u}-\boldsymbol{u}_{h}\right\|_{F}^{2}\right)^{1 / 2} \\
& \quad \lesssim \alpha^{-1 / 2}\left\|\boldsymbol{u}-\boldsymbol{u}_{h}\right\|_{\text {en }}+\gamma_{0}\left(\sum_{F \in \mathcal{F}_{h}^{C}} \frac{1}{h_{F}}\left\|\boldsymbol{u}-\boldsymbol{u}_{h}\right\|_{F}^{2}\right)^{1 / 2} \cdot
\end{aligned}
$$

Combining this bound with (3.22), we obtain (3.23).

\section{Identification of the error components}

We consider the resolution of the (nonlinear) discrete problem (2.7) with an iterative method in which, at each iteration $k \geq 1$, the nonlinear term $\left[P_{1, \gamma}^{n}(\cdot)\right]_{\mathbb{R}^{-}}$is replaced by a linear approximation $P_{\text {lin }}^{k-1}(\cdot)$. A new approximation of the discrete solution is then obtained solving the following problem: Find $\boldsymbol{u}_{h}^{k} \in \boldsymbol{V}_{h}$ such that

$$
a\left(\boldsymbol{u}_{h}^{k}, \boldsymbol{v}_{h}\right)-\left(P_{\operatorname{lin}}^{k-1}\left(\boldsymbol{u}_{h}^{k}\right), v_{h}^{n}\right)_{\Gamma_{C}}=L\left(\boldsymbol{v}_{h}\right) \quad \forall \boldsymbol{v}_{h} \in \boldsymbol{V}_{h} .
$$

The linearized operator $P_{\operatorname{lin}}^{k-1}(\cdot)$ is based on the following regularization of the projection $[\cdot]_{\mathbb{R}^{-}}$: Given a real number $\delta>0$ (representing the amount of regularization),

$$
[x]_{\mathrm{reg}, \delta}:= \begin{cases}x & \text { if } x \leq-\delta \\ -\frac{1}{4 \delta} x^{2}+\frac{1}{2} x-\frac{\delta}{4} & \text { if }|x|<\delta \\ 0 & \text { if } x \geq \delta .\end{cases}
$$




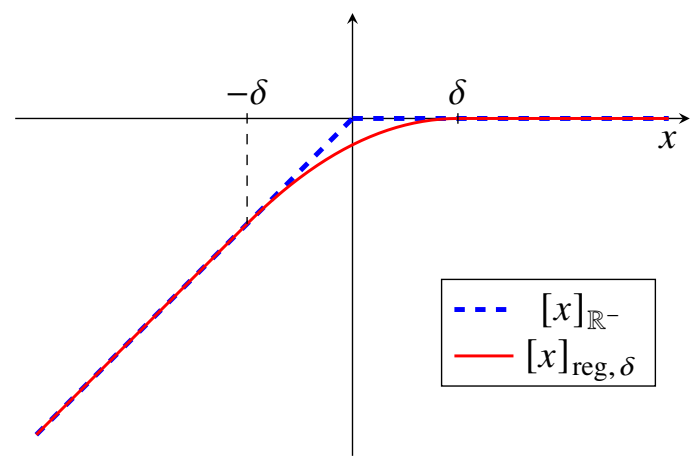

Figure 2: Comparison between the projection operator $[x]_{\mathbb{R}^{-}}$(blue) and the regularized operator $[x]_{\mathrm{reg}, \delta}(\mathrm{red})$.

Figure 2 shows the graphs of the projection operator $[\cdot]_{\mathbb{R}^{-}}$and of the regularized operator $[\cdot]_{\text {reg, },}$. Notice that they coincide for $|x| \geq \delta$ and $[\cdot]_{\mathrm{reg}, \delta}$ belongs to $C^{1}(\mathbb{R})$ (but not to $C^{2}(\mathbb{R})$ ). The linearized operator $P_{\operatorname{lin}}^{k-1}(\cdot)$ is obtained setting, for any $\boldsymbol{w}_{h} \in \boldsymbol{V}_{h}$,

$$
\begin{aligned}
P_{\mathrm{lin}, \delta}^{k-1}\left(\boldsymbol{w}_{h}\right) & :=\left[P_{1, \gamma}^{n}\left(\boldsymbol{u}_{h}^{k-1}\right)\right]_{\mathrm{reg}, \delta}+\left.\frac{\partial\left[P_{1, \gamma}^{n}(\boldsymbol{v})\right]_{\mathrm{reg}, \delta}}{\partial \boldsymbol{v}}\right|_{\boldsymbol{v}=\boldsymbol{u}_{h}^{k-1}} \cdot\left(\boldsymbol{w}_{h}-\boldsymbol{u}_{h}^{k-1}\right) \\
& =\left[P_{1, \gamma}^{n}\left(\boldsymbol{u}_{h}^{k-1}\right)\right]_{\mathrm{reg}, \delta}+\left.\frac{\mathrm{d}[x]_{\mathrm{reg}, \delta}}{\mathrm{d} x}\right|_{x=P_{1, \gamma}^{n}\left(\boldsymbol{u}_{h}^{k-1}\right)}\left(P_{1, \gamma}^{n}\left(\boldsymbol{w}_{h}\right)-P_{1, \gamma}^{n}\left(\boldsymbol{u}_{h}^{k-1}\right)\right) .
\end{aligned}
$$

Here, we add the subscript $\delta$ to emphasize that the linear operator depends on the choice of this parameter. The refined error estimate presented in the following section enables an automatic tuning of $\delta$.

\subsection{A posteriori error estimate distinguishing the error components}

We present in this section an error estimate for $\boldsymbol{u}_{h}^{k}$ which enables one to identify and separate the different components of the error. The estimate hinges on the following assumption:

Assumption 10 (Decomposition of the stress reconstruction). Let $\sigma_{h}^{k}$ be an equilibrated stress reconstruction in the sense of Definition 3. Then, $\sigma_{h}^{k}$ can be decomposed into three parts

$$
\sigma_{h}^{k}=\sigma_{h, \text { dis }}^{k}+\sigma_{h, \text { reg }}^{k}+\sigma_{h, \text { lin }}^{k}
$$

where $\sigma_{h \text {,dis }}^{k}$ represents discretization, $\sigma_{h, \text { reg }}^{k}$ represents regularization, and $\sigma_{h, \text { lin }}^{k}$ represents linearization.

In Section 5 we will show how to obtain an equilibrated stress reconstruction which satisfies this 
assumption. Finally, we introduce the following local error estimators: For every element $T \in \mathcal{T}_{h}$,

$$
\begin{aligned}
& \eta_{\mathrm{osc}, T}^{k}:=\frac{h_{T}}{\pi}\left\|\boldsymbol{f}+\operatorname{div} \sigma_{h}^{k}\right\|_{T}, \\
& \eta_{\mathrm{str}, T}^{k}:=\left\|\boldsymbol{\sigma}_{h, \mathrm{dis}}^{k}-\boldsymbol{\sigma}\left(\boldsymbol{u}_{h}^{k}\right)\right\|_{T}, \\
& \eta_{\mathrm{reg} 1, T}^{k}:=\left\|\sigma_{h, \text { reg }}^{k}\right\|_{T} \quad \text { and } \quad \eta_{\mathrm{reg} 2, T}^{k}:=\sum_{F \in \mathcal{F}_{T}^{C}} h_{F}^{1 / 2}\left\|\sigma_{h, \text { reg }}^{k, n}\right\|_{F}, \\
& \eta_{\operatorname{lin} 1, T}^{k}:=\left\|\sigma_{h, \operatorname{lin}}^{k}\right\|_{T} \quad \text { and } \quad \eta_{\operatorname{lin} 2, T}^{k}:=\sum_{F \in \mathcal{F}_{T}^{C}} h_{F}^{1 / 2}\left\|\sigma_{h, \operatorname{lin}}^{k, n}\right\|_{F}, \quad \text { (linearization) } \\
& \eta_{\mathrm{Neu}, T}^{k}:=\sum_{F \in \mathcal{F}_{T}^{N}} C_{t, T, F} h_{F}^{1 / 2}\left\|\boldsymbol{g}_{N}-\boldsymbol{\sigma}_{h}^{k} \boldsymbol{n}\right\|_{F}, \\
& \eta_{\mathrm{cnt}, \mathrm{T}}^{k}:=\sum_{F \in \mathcal{F}_{T}^{C}} h_{F}^{1 / 2}\left\|\left[P_{1, \gamma}^{n}\left(\boldsymbol{u}_{h}^{k}\right)\right]_{\mathbb{R}^{-}}-\sigma_{h, \mathrm{dis}}^{k, n}\right\|_{F} .
\end{aligned}
$$

The corresponding global error estimators are defined setting

$$
\eta_{\bullet}^{k}:=\left(\sum_{T \in \mathcal{T}_{h}}\left(\eta_{\bullet, T}^{k}\right)^{2}\right)^{1 / 2} .
$$

Theorem 11 (A posteriori error estimate distinguishing the error components). Let $\boldsymbol{u}_{h}^{k} \in \boldsymbol{V}_{h}$ be the solution of the linearized problem (4.1) with $P_{\text {lin, } \delta}(\cdot)$ defined by (4.2), and let $\mathcal{R}\left(\boldsymbol{u}_{h}^{k}\right)$ be the residual of $\boldsymbol{u}_{h}^{k}$ defined by (3.1). Then, under Assumption 10, it holds

$$
\begin{aligned}
& \|\| \mathcal{R}\left(\boldsymbol{u}_{h}^{k}\right) \|_{*} \\
& \quad \leq\left[\sum_{T \in \mathcal{T}_{h}}\left(\left(\eta_{\mathrm{osc}, \mathrm{T}}^{k}+\eta_{\mathrm{str}, \mathrm{T}}^{k}+\eta_{\mathrm{reg} 1, \mathrm{~T}}^{k}+\eta_{\mathrm{lin} 1, \mathrm{~T}}^{k}+\eta_{\mathrm{Neu}, \mathrm{T}}^{k}\right)^{2}+\left(\eta_{\mathrm{cnt}, \mathrm{T}}^{k}+\eta_{\mathrm{reg} 2, \mathrm{~T}}^{k}+\eta_{\mathrm{lin} 2, \mathrm{~T}}^{k}\right)^{2}\right)\right]^{1 / 2}
\end{aligned}
$$

and, as a result,

$$
\left\|\mathcal{R}\left(\boldsymbol{u}_{h}^{k}\right)\right\|_{*} \leq\left[\left(\eta_{\mathrm{osc}}^{k}+\eta_{\mathrm{str}}^{k}+\eta_{\mathrm{reg} 1}^{k}+\eta_{\mathrm{lin} 1}^{k}+\eta_{\mathrm{Neu}}^{k}\right)^{2}+\left(\eta_{\mathrm{cnt}}^{k}+\eta_{\mathrm{reg} 2}^{k}+\eta_{\mathrm{lin} 2}^{k}\right)^{2}\right]^{1 / 2} .
$$

Proof. Proceeding as in the proof of Theorem 4, we immediately get

$$
\begin{aligned}
& \left\|\mathcal{R}\left(\boldsymbol{u}_{h}^{k}\right)\right\|_{*} \\
& \leq\left[\sum_{T \in \mathcal{T}_{h}}\left(\left(\eta_{\mathrm{osc}, T}^{k}+\left\|\boldsymbol{\sigma}_{h}^{k}-\sigma\left(\boldsymbol{u}_{h}^{k}\right)\right\|_{T}+\eta_{\mathrm{Neu}, T}^{k}\right)^{2}+\left(\sum_{F \in \mathcal{F}_{T}^{C}} h_{F}^{1 / 2}\left\|\left[P_{1, \gamma}^{n}\left(\boldsymbol{u}_{h}^{k}\right)\right]_{\mathbb{R}^{-}}-\sigma_{h}^{k, n}\right\|_{F}\right)^{2}\right)\right]^{1 / 2} .
\end{aligned}
$$

Decomposing $\sigma_{h}^{k}$ according to (4.3) and using the triangle inequality, we arrive at (4.6). Finally, (4.7) is obtained from (4.6) applying twice the inequality $\sum_{T \in \mathcal{T}_{h}}\left(\sum_{i=1}^{m} a_{i, T}\right)^{2} \leq\left(\sum_{i=1}^{m} a_{i}\right)^{2}$ valid for all families of nonnegative real numbers $\left(a_{i, T}\right)_{1 \leq i \leq m, T \in \mathcal{T}_{h}}$ with $a_{i}:=\left(\sum_{T \in \mathcal{T}_{h}} a_{i, T}^{2}\right)^{1 / 2}$ for all $1 \leq i \leq m$. 


\subsection{Fully adaptive algorithm}

We propose an adaptive algorithm based on the error estimators (4.4) and (4.5), and on the result of Theorem 11. Denote by $\gamma_{\text {reg }}, \gamma_{\text {lin }} \in(0,1)$ two user-dependent parameters that represent the relative magnitude of the regularization and linearization errors with respect to the total error. Moreover, we define the following local estimators:

$$
\eta_{\mathrm{reg}, T}^{k}:=\eta_{\mathrm{reg} 1, T}^{k}+\eta_{\mathrm{reg} 2, T}^{k}, \quad \eta_{\operatorname{lin}, T}^{k}:=\eta_{\operatorname{lin} 1, T}^{k}+\eta_{\operatorname{lin} 2, T}^{k} .
$$

The corresponding global counterparts are given by (4.5) with $\bullet \in\{\mathrm{reg}$, lin $\}$. With these estimators and the parameters $\gamma_{\mathrm{reg}}, \gamma_{\text {lin }}$, we define stopping criteria for the regularization and linearization loops, respectively, so that both the parameter $\delta$ and the number of Newton iterations on every mesh refinement iteration will be fixed automatically by the adaptive algorithm. For all $T \in \mathcal{T}_{h}$, the total error estimator is given by

$$
\eta_{\mathrm{tot}, T}^{k}:=\left[\left(\eta_{\mathrm{osc}, T}^{k}+\eta_{\mathrm{str}, T}^{k}+\eta_{\mathrm{reg} 1, T}^{k}+\eta_{\mathrm{lin} 1, T}^{k}+\eta_{\mathrm{Neu}, T}^{k}\right)^{2}+\left(\eta_{\mathrm{cnt}, T}^{k}+\eta_{\mathrm{reg} 2, T}^{k}+\eta_{\mathrm{lin} 2, T}^{k}\right)^{2} \cdot\right]^{1 / 2}
$$

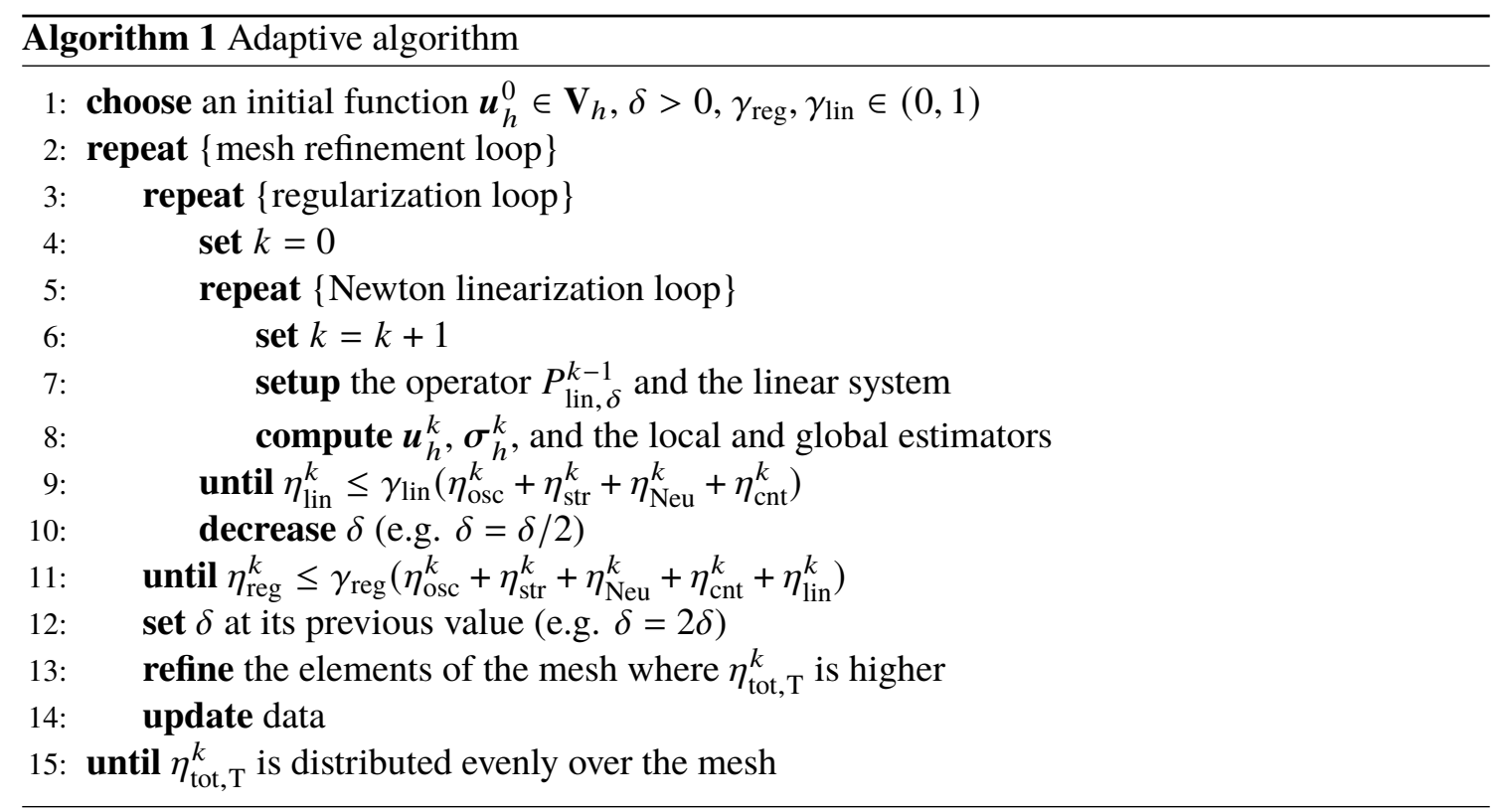

Remark 12 (Local stopping criteria). The stopping critera in Lines 9 and 11 can alternatively be enforced locally inside each element:

$$
\begin{array}{ll}
\eta_{\text {lin, } \mathrm{T}}^{k} \leq \gamma_{\mathrm{lin}, \mathrm{T}}\left(\eta_{\mathrm{osc}, \mathrm{T}}^{k}+\eta_{\mathrm{str}, \mathrm{T}}^{k}+\eta_{\mathrm{Neu}, \mathrm{T}}^{k}+\eta_{\mathrm{cnt}, \mathrm{T}}^{k}\right) & \forall T \in \mathcal{T}_{h}, \\
\eta_{\mathrm{reg}, \mathrm{T}}^{k} \leq \gamma_{\mathrm{reg}, \mathrm{T}}\left(\eta_{\mathrm{osc}, \mathrm{T}}^{k}+\eta_{\mathrm{str}, \mathrm{T}}^{k}+\eta_{\mathrm{Neu}, \mathrm{T}}^{k}+\eta_{\mathrm{cnt}, \mathrm{T}}^{k}+\eta_{\mathrm{lin}, \mathrm{T}}^{k}\right) & \forall T \in \mathcal{T}_{h},
\end{array}
$$

where the parameters $\gamma_{\mathrm{lin}, \mathrm{T}}, \gamma_{\mathrm{reg}, \mathrm{T}} \in(0,1)$ can possibly vary element by element; see, e.g., [26] and also the discussion in [19, Section 4.1].

\section{Equilibrated stress reconstructions}

We first show how to construct an equilibrated stress reconstruction $\sigma_{h}$ that satisfies the conditions of Definition 3, then modify the construction to match Assumption 10. 

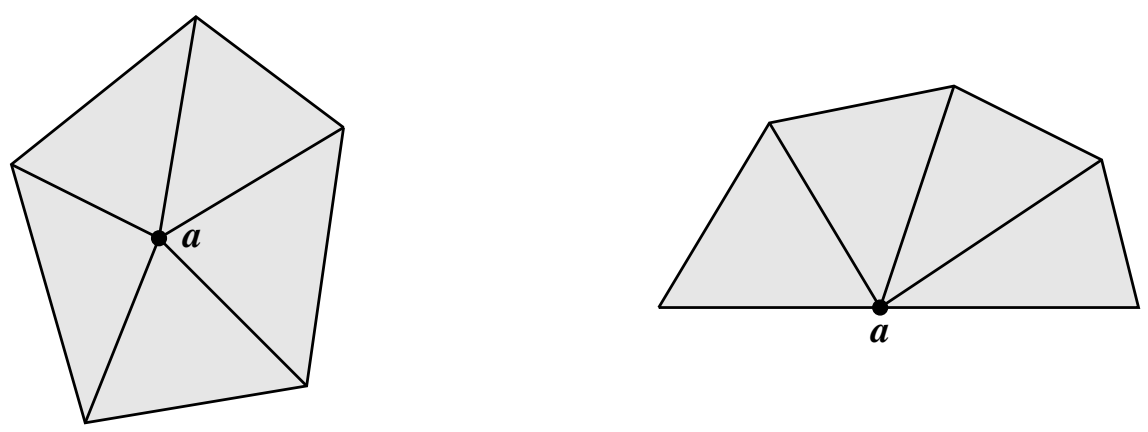

Figure 3: Illustration of a patch $\omega_{\boldsymbol{a}}$ around an inner node $\boldsymbol{a} \in \mathcal{V}_{h}^{i}$ (left), and around a boundary node $\boldsymbol{a} \in \mathcal{V}_{h}^{b}($ right $)$.

\subsection{Basic equilibrated stress reconstruction}

Following the path of [5], we construct $\sigma_{h}$ patchwise around the mesh vertices using ArnoldFalk-Winther mixed finite element spaces [1], which are based on a stress tensor constructed in the Brezzi-Douglas-Marini space (see, e.g., [6, Chapter 3] and [4, Chapter 2]), along with Lagrange multipliers that enforce a weak symmetry constraint. From this point on, $\mathcal{V}_{h}^{D}$ will denote the set collecting all mesh vertices which lie on some Dirichlet boundary face. Notice that $\mathcal{V}_{h}^{D}$ also contains the vertices lying at the intersection between $\Gamma_{D}$ and $\Gamma_{\bullet}, \bullet \in\{N, C\}$.

For any element $T \in \mathcal{T}_{h}$, and any integer $q \geq 1$, we set

$$
\Sigma_{T}:=\mathbb{P}^{q}(T), \quad \boldsymbol{U}_{T}:=\mathcal{P}^{q-1}(T), \quad \boldsymbol{\Lambda}_{T}:=\left\{\boldsymbol{\mu} \in \mathbb{P}^{q-1}(T): \boldsymbol{\mu}=-\boldsymbol{\mu}^{T}\right\} .
$$

At the global level, we define the following spaces

$$
\begin{aligned}
\boldsymbol{\Sigma}_{h} & :=\left\{\tau_{h} \in \mathbb{H}(\operatorname{div}, \Omega):\left.\tau_{h}\right|_{T} \in \boldsymbol{\Sigma}_{T} \text { for any } T \in \mathcal{T}_{h}\right\}, \\
\boldsymbol{U}_{h} & :=\left\{\boldsymbol{v}_{h} \in \boldsymbol{L}^{2}(\Omega):\left.\boldsymbol{v}_{h}\right|_{T} \in \boldsymbol{U}_{T} \text { for any } T \in \mathcal{T}_{h}\right\}, \\
\boldsymbol{\Lambda}_{h} & :=\left\{\boldsymbol{\mu}_{h} \in \mathbb{L}^{2}(\Omega):\left.\boldsymbol{\mu}_{h}\right|_{T} \in \boldsymbol{\Lambda}_{T} \text { for any } T \in \mathcal{T}_{h}\right\} .
\end{aligned}
$$

Notice that $\boldsymbol{\Sigma}_{h} \subset \mathbb{H}(\mathbf{d i v}, \Omega)$ implies that its elements have continuous normal components across interfaces [15, Lemma 1.17]. Now, let $q=p$, let $\boldsymbol{u}_{h}$ be the solution of (2.7), and fix a mesh vertex $\boldsymbol{a}$. We denote by $\omega_{\boldsymbol{a}}$ the patch around the node $\boldsymbol{a}$, see Figure 3, by $\boldsymbol{n}_{\omega_{\boldsymbol{a}}}$ the normal unit outward vector on its boundary $\partial \omega_{\boldsymbol{a}}$ and by $\psi_{\boldsymbol{a}}$ the hat function associated with $\boldsymbol{a}$. On any patch $\omega_{\boldsymbol{a}}$ we then define the following spaces:

$$
\begin{aligned}
& \boldsymbol{\Sigma}_{h}^{\boldsymbol{a}}:=\left\{\begin{array}{lll}
\left\{\boldsymbol{\tau}_{h} \in \boldsymbol{\Sigma}_{h}\left(\omega_{\boldsymbol{a}}\right): \boldsymbol{\tau}_{h} \boldsymbol{n}_{\omega_{\boldsymbol{a}}}=\mathbf{0} \text { on } \partial \omega_{\boldsymbol{a}} \backslash \Gamma_{D}\right\} & \text { if } \boldsymbol{a} \in \mathcal{V}_{h}^{b} \\
\left\{\boldsymbol{\tau}_{h} \in \boldsymbol{\Sigma}_{h}\left(\omega_{\boldsymbol{a}}\right): \boldsymbol{\tau}_{h} \boldsymbol{n}_{\omega_{\boldsymbol{a}}}=\mathbf{0} \text { on } \partial \omega_{\boldsymbol{a}}\right\} & \text { otherwise, }
\end{array}\right. \\
& \boldsymbol{\Sigma}_{h, N, C}^{\boldsymbol{a}}:=\left\{\begin{array}{cc}
\left\{\boldsymbol{\tau}_{h} \in \boldsymbol{\Sigma}_{h}\left(\omega_{\boldsymbol{a}}\right): \boldsymbol{\tau}_{h} \boldsymbol{n}_{\omega_{\boldsymbol{a}}}=\mathbf{0} \text { on } \partial \omega_{\boldsymbol{a}} \backslash \partial \Omega,\right. & \\
\boldsymbol{\tau}_{h} \boldsymbol{n}_{\omega_{\boldsymbol{a}}}=\prod_{\boldsymbol{\Sigma}_{h} \boldsymbol{n}_{\omega_{\boldsymbol{a}}}\left(\psi_{\boldsymbol{a}} \boldsymbol{g}_{N}\right) \text { on } \partial \omega_{\boldsymbol{a}} \cap \Gamma_{N}, \text { and }} \boldsymbol{\tau}_{h} \boldsymbol{n}_{\omega_{\boldsymbol{a}}}=\prod_{\left.\boldsymbol{\Sigma}_{h} \boldsymbol{n}_{\omega_{\boldsymbol{a}}}\left(\psi_{\boldsymbol{a}}\left[P_{1, \gamma}^{n}\left(\boldsymbol{u}_{h}\right)\right]_{\mathbb{R}^{-}} \boldsymbol{n}\right) \text { on } \partial \omega_{\boldsymbol{a}} \cap \Gamma_{C}\right\},} & \text { if } \boldsymbol{a} \in \mathcal{V}_{h}^{b} \\
\boldsymbol{\Sigma}_{h}^{\boldsymbol{a}} & \text { otherwise }
\end{array}\right. \\
& \boldsymbol{U}_{h}^{\boldsymbol{a}}:= \begin{cases}\boldsymbol{U}_{h}\left(\omega_{\boldsymbol{a}}\right) & \text { if } \boldsymbol{a} \in \mathcal{V}_{h}^{D} \\
\left\{\boldsymbol{v}_{h} \in \boldsymbol{U}_{h}\left(\omega_{\boldsymbol{a}}\right):\left(\boldsymbol{v}_{h}, \boldsymbol{z}\right)_{\omega_{\boldsymbol{a}}}=0 \text { for any } \boldsymbol{z} \in \boldsymbol{R} \boldsymbol{M}^{d}\right\} & \text { otherwise, }\end{cases} \\
& \boldsymbol{\Lambda}_{h}^{a}:=\Lambda_{h}\left(\omega_{a}\right) .
\end{aligned}
$$

Above, $\boldsymbol{\Sigma}_{h}\left(\omega_{\boldsymbol{a}}\right), \boldsymbol{U}_{h}\left(\omega_{\boldsymbol{a}}\right)$, and $\boldsymbol{\Lambda}_{h}\left(\omega_{\boldsymbol{a}}\right)$ denote the restrictions of the spaces $\boldsymbol{\Sigma}_{h}, \boldsymbol{U}_{h}$ and $\boldsymbol{\Lambda}_{h}$ to the subdomain $\omega_{\boldsymbol{a}}$, respectively. Moreover, $\boldsymbol{\Sigma}_{h} \boldsymbol{n}_{\omega_{\boldsymbol{a}}}$ is the space of normal traces on the patch boundary 
$\partial \omega_{\boldsymbol{a}}$ of elements in $\boldsymbol{\Sigma}_{h}\left(\omega_{\boldsymbol{a}}\right)$, i.e., it is the space of vector-valued broken polynomials of total degree $\leq p$ on the set of boundary faces of the patch, while $\boldsymbol{R} \boldsymbol{M}^{d}$ is space of rigid-body motions, i.e., $\boldsymbol{R} \boldsymbol{M}^{2}:=\left\{\boldsymbol{b}+c\left(x_{2},-x_{1}\right)^{\top}: \boldsymbol{b} \in \mathbb{R}^{2}, c \in \mathbb{R}\right\}$ and $\boldsymbol{R} \boldsymbol{M}^{3}:=\left\{\boldsymbol{b}+\boldsymbol{c} \times \boldsymbol{x}: \boldsymbol{b}, \boldsymbol{c} \in \mathbb{R}^{3}\right\}$.

Remark 13 (Boundary condition for the reconstruction on internal vertices). In the definition (5.1) of $\boldsymbol{\Sigma}_{h}^{\boldsymbol{a}}$, we distinguish between boundary and internal vertices in order to ensure, in the case $\boldsymbol{a} \in \mathcal{V}_{h}^{i}$, zero normal components on the whole boundary of the patch $\omega_{\boldsymbol{a}}$ even if $\left|\partial \omega_{\boldsymbol{a}} \cap \Gamma_{D}\right|>0$.

Construction 14 (Basic equilibrated stress reconstruction). Let, for any vertex $\boldsymbol{a} \in \mathcal{V}_{h},\left(\sigma_{h}^{a}, \boldsymbol{r}_{h}^{\boldsymbol{a}}, \lambda_{h}^{\boldsymbol{a}}\right) \in$ $\Sigma_{h, N, C}^{a} \times U_{h}^{a} \times \Lambda_{h}^{a}$ be the solution to the following problem:

$$
\begin{aligned}
\left(\sigma_{h}^{a}, \tau_{h}\right)_{\omega_{\boldsymbol{a}}}+\left(\boldsymbol{r}_{h}^{a}, \operatorname{div} \tau_{h}\right)_{\omega_{\boldsymbol{a}}}+\left(\lambda_{h}^{\boldsymbol{a}}, \tau_{h}\right)_{\omega_{\boldsymbol{a}}} & =\left(\psi_{\boldsymbol{a}} \sigma\left(\boldsymbol{u}_{h}\right), \tau_{h}\right)_{\omega_{\boldsymbol{a}}} & & \forall \tau_{h} \in \Sigma_{h}^{a}, \\
\left(\operatorname{div} \sigma_{h}^{a}, \boldsymbol{v}_{h}\right)_{\omega_{\boldsymbol{a}}} & =\left(-\psi_{\boldsymbol{a}} f+\sigma\left(\boldsymbol{u}_{h}\right) \nabla \psi_{\boldsymbol{a}}, v_{h}\right)_{\omega_{\boldsymbol{a}}} & & \forall v_{h} \in \boldsymbol{U}_{h}^{\boldsymbol{a}}, \\
\left(\boldsymbol{\sigma}_{h}^{\boldsymbol{a}}, \boldsymbol{\mu}_{h}\right)_{\omega_{\boldsymbol{a}}} & =0 & & \forall \boldsymbol{\mu}_{h} \in \Lambda_{h}^{\boldsymbol{a}} .
\end{aligned}
$$

Extending $\boldsymbol{\sigma}_{h}^{a}$ by zero outside the patch $\omega_{\boldsymbol{a}}$, we set $\boldsymbol{\sigma}_{h}:=\sum_{\boldsymbol{a} \in \mathcal{V}_{h}} \boldsymbol{\sigma}_{h}^{a}$.

By definition of the space $\Sigma_{h, N, C}^{a}$, a homogeneous Neumann boundary condition is enforced on the whole boundary of $\omega_{\boldsymbol{a}}$ for interior vertices and on $\partial \omega_{\boldsymbol{a}} \backslash \partial \Omega$ for boundary vertices. In particular, for boundary vertices in $\mathcal{V}_{h}^{b} \backslash \mathcal{V}_{h}^{D}$, a possibly non homogeneous Neumann boundary condition is enforced on the boundary faces of the patch. Therefore, when $a \in \mathcal{V}_{h}^{i}$ or $a \in \mathcal{V}_{h}^{b} \backslash \mathcal{V}_{h}^{D}$, the right hand side of (5.3b) has to verify the following Neumann compatibility condition:

$$
\begin{aligned}
&\left(-\psi_{\boldsymbol{a}} \boldsymbol{f}+\boldsymbol{\sigma}\left(\boldsymbol{u}_{h}\right) \boldsymbol{\nabla} \psi_{\boldsymbol{a}}, z\right)_{\omega_{\boldsymbol{a}}} \\
& \quad=\left(\Pi_{\Sigma_{h} \boldsymbol{n}_{\omega \boldsymbol{a}}}\left(\psi_{\boldsymbol{a}} \boldsymbol{g}_{N}\right), z\right)_{\partial \omega_{\boldsymbol{a}} \cap \Gamma_{N}}+\left(\Pi_{\Sigma_{h} \boldsymbol{n}_{\omega \boldsymbol{a}}}\left(\psi_{\boldsymbol{a}}\left[P_{1, \gamma}^{n}\left(\boldsymbol{u}_{h}\right)\right]_{\mathbb{R}^{-}} \boldsymbol{n}\right), z\right)_{\partial \omega_{\boldsymbol{a}} \cap \Gamma_{C}}
\end{aligned}
$$

for any $z \in \boldsymbol{R} \boldsymbol{M}^{d}$. Fixing a rigid-body motion $z$, it is possible to check that (5.4) holds by taking $\psi_{\boldsymbol{a}} z$ as test function in (2.7). The following Lemma lists the main properties of the tensor $\sigma_{h}$ resulting from Construction 14. In particular, it shows that $\sigma_{h}$ satisfies all the conditions of Definition 3, i.e., it is an equilibrated stress reconstruction.

Lemma 15 (Properties of $\sigma_{h}$ ). Let $\sigma_{h}$ be defined by Construction 14. Then, it holds

1. $\sigma_{h} \in \mathbb{H}(\operatorname{div}, \Omega)$;

2. For every $T \in \mathcal{T}_{h}$ and every $\boldsymbol{v}_{T} \in \mathcal{P}^{p-1}(T)$, $\left(\boldsymbol{d i v} \sigma_{h}+\boldsymbol{f}, \boldsymbol{v}_{T}\right)_{T}=0$;

3. For every $F \in \mathcal{F}_{h}^{N}$ and every $\boldsymbol{v}_{F} \in \mathcal{P}^{p}(F),\left(\boldsymbol{\sigma}_{h} \boldsymbol{n}, \boldsymbol{v}_{F}\right)_{F}=\left(\boldsymbol{g}_{N}, \boldsymbol{v}_{F}\right)_{F}$;

4. For every $F \in \mathcal{F}_{h}^{C}$ and every $\boldsymbol{v}_{F} \in \mathcal{P}^{p}(F)$,

$$
\left(\boldsymbol{\sigma}_{h} \boldsymbol{n}, \boldsymbol{v}_{F}\right)_{F}=\left(\left[P_{1, \gamma}^{n}\left(\boldsymbol{u}_{h}\right)\right]_{\mathbb{R}^{-}} \boldsymbol{n}, \boldsymbol{v}_{F}\right)_{F}=\left(\left[P_{1, \gamma}^{n}\left(\boldsymbol{u}_{h}\right)\right]_{\mathbb{R}^{-}}, v_{F}^{n}\right)_{F} .
$$

Proof. 1) By definition, $\sigma_{h}^{\boldsymbol{a}} \in \mathbb{H}\left(\mathbf{d i v}, \omega_{\boldsymbol{a}}\right)$ for any $\boldsymbol{a} \in \mathcal{V}_{h}$. Due to the no-flux boundary condition on internal faces enforced in the local problem on $\omega_{\boldsymbol{a}}$, the extension of $\sigma_{h}^{a}$ by zero outside the patch is in $\mathbb{H}(\mathbf{d i v}, \Omega)$ and, as a consequence, $\sigma_{h} \in \mathbb{H}(\mathbf{d i v}, \Omega)$.

2) First, we check that, for any $\boldsymbol{a} \in \mathcal{V}_{h}$, equation (5.3b) holds for every $\boldsymbol{v}_{h} \in \boldsymbol{U}_{h}\left(\omega_{\boldsymbol{a}}\right)$. If $\boldsymbol{a} \in \mathcal{V}_{h}^{D}$, this is trivial since $\boldsymbol{U}_{h}^{\boldsymbol{a}}=\boldsymbol{U}_{h}\left(\omega_{\boldsymbol{a}}\right)$. If, on the other hand, $\boldsymbol{a} \in \mathcal{V}_{h} \backslash \mathcal{V}_{h}^{D}$, it is sufficient to use the fact that $\boldsymbol{U}_{h}^{\boldsymbol{a}}=\left(\boldsymbol{R} \boldsymbol{M}^{d}\right)^{\perp}$ (with orthogonal taken with respect to the $\boldsymbol{L}^{2}\left(\omega_{\boldsymbol{a}}\right)$-product) along with the Green formula, the definition (5.2) of $\boldsymbol{\Sigma}_{h}^{\boldsymbol{a}}$, the Neumann compatibility condition (5.4), and (5.3c). 
Now, fix $T \in \mathcal{T}_{h}$ and let $\boldsymbol{v}_{T} \in \mathcal{P}^{p-1}(T)$. Extending $\boldsymbol{v}_{T}$ by zero outside of $T$, we have $\boldsymbol{v}_{T} \in \boldsymbol{U}_{h}\left(\omega_{\boldsymbol{a}}\right)$ for all $\boldsymbol{a} \in \mathcal{V}_{T}$. Indeed, by definition, $\boldsymbol{U}_{h}\left(\omega_{\boldsymbol{a}}\right)$ is composed by piecewise polynomials of degree at most $p-1$ that can be chosen independently inside each element of the patch. Summing (5.3b) over $a \in \mathcal{V}_{T}$ we obtain:

$$
0=\sum_{\boldsymbol{a} \in \mathcal{V}_{T}}\left[\left(\operatorname{div} \boldsymbol{\sigma}_{h}^{\boldsymbol{a}}, \boldsymbol{v}_{T}\right)_{\omega_{\boldsymbol{a}}}+\left(\psi_{\boldsymbol{a}} \boldsymbol{f}, \boldsymbol{v}_{T}\right)_{\omega_{\boldsymbol{a}}}-\left(\boldsymbol{\sigma}\left(\boldsymbol{u}_{h}\right) \boldsymbol{\nabla} \psi_{\boldsymbol{a}}, \boldsymbol{v}_{T}\right)_{\omega_{\boldsymbol{a}}}\right]=\left(\operatorname{div} \boldsymbol{\sigma}_{h}+\boldsymbol{f}, \boldsymbol{v}_{T}\right)_{T}
$$

Here, we have used the fact that $\left.\sigma_{h}\right|_{T}=\left.\sum_{\boldsymbol{a} \in \mathcal{V}_{T}} \boldsymbol{\sigma}_{h}^{\boldsymbol{a}}\right|_{T}$ and $\sum_{\boldsymbol{a} \in \mathcal{V}_{T}} \psi_{\boldsymbol{a}}=1$ over $T$ (so that, in particular, $\sum_{\boldsymbol{a} \in \mathcal{V}_{T}} \boldsymbol{\nabla} \psi_{\boldsymbol{a}} \equiv 0$ ).

3,4) We only detail the proof of 3) as that of 4) is similar. Let $F \in \mathcal{F}_{h}^{N}$ and let $\boldsymbol{v}_{F}$ be a polynomial defined on $F$ from the discrete normal trace space $\left.\left(\boldsymbol{\Sigma}_{h} \boldsymbol{n}\right)\right|_{F}$, i.e., a polynomial of total degree at most $p$. Then, by the definition of $\Sigma_{h, N, C}^{a}(5.2)$,

$$
\left(\boldsymbol{\sigma}_{h} \boldsymbol{n}, \boldsymbol{v}_{F}\right)_{F}=\sum_{\boldsymbol{a} \in \mathcal{V}_{F}}\left(\boldsymbol{\sigma}_{h}^{\boldsymbol{a}} \boldsymbol{n}, \boldsymbol{v}_{F}\right)_{F}=\sum_{\boldsymbol{a} \in \mathcal{V}_{F}}\left(\psi_{\boldsymbol{a}} \boldsymbol{g}_{N}, \boldsymbol{v}_{F}\right)_{F}=\left(\boldsymbol{g}_{N}, \boldsymbol{v}_{F}\right)_{F} .
$$

\subsection{Stress reconstruction distinguishing the error components}

In Construction 14 we used the solution $\boldsymbol{u}_{h}$ of the nonlinear problem (2.7) to reconstruct an equilibrated stress $\sigma_{h}$. However, as argued in Section 4, in practice we only dispose of an approximated solution obtained by means of a linearization method. Let $k \geq 1$ be an integer and let $\boldsymbol{u}_{h}^{k}$ be the solution of the linearized problem (4.1) with operator $P_{\operatorname{lin}, \delta}(\cdot)$ defined by (4.2). Then, for any boundary vertex $\boldsymbol{a} \in \mathcal{V}_{h}^{b}$, we set

$$
\begin{aligned}
& \boldsymbol{\Sigma}_{h, N, C, \mathrm{dis}}^{\boldsymbol{a}, k}:=\left\{\boldsymbol{\tau}_{h} \in \boldsymbol{\Sigma}_{h}\left(\omega_{\boldsymbol{a}}\right): \boldsymbol{\tau}_{h} \boldsymbol{n}_{\omega_{\boldsymbol{a}}}=\mathbf{0} \text { on } \partial \omega_{\boldsymbol{a}} \backslash \partial \Omega,\right. \\
& \boldsymbol{\tau}_{h} \boldsymbol{n}_{\omega_{\boldsymbol{a}}}=\Pi_{\boldsymbol{\Sigma}_{h} \boldsymbol{n}_{\omega_{\boldsymbol{a}}}}\left(\psi_{\boldsymbol{a}} \boldsymbol{g}_{N}\right) \text { on } \partial \omega_{\boldsymbol{a}} \cap \Gamma_{N} \text { and } \\
& \left.\boldsymbol{\tau}_{h} \boldsymbol{n}_{\omega_{\boldsymbol{a}}}=\Pi_{\boldsymbol{\Sigma}_{h} \boldsymbol{n}_{\omega_{\boldsymbol{a}}}}\left(\psi_{\boldsymbol{a}}\left[P_{1, \gamma}^{n}\left(\boldsymbol{u}_{h}^{k}\right)\right]_{\mathbb{R}^{-}} \boldsymbol{n}\right) \text { on } \partial \omega_{\boldsymbol{a}} \cap \Gamma_{C}\right\}, \\
& \boldsymbol{\Sigma}_{h, N, C, \text { reg }}^{\boldsymbol{a}, k}:=\left\{\boldsymbol{\tau}_{h} \in \boldsymbol{\Sigma}_{h}\left(\omega_{\boldsymbol{a}}\right): \boldsymbol{\tau}_{h} \boldsymbol{n}_{\omega_{\boldsymbol{a}}}=\mathbf{0} \text { on } \partial \omega_{\boldsymbol{a}} \backslash \partial \Omega \text { and on } \partial \omega_{\boldsymbol{a}} \cap \Gamma_{N}\right. \text {, and } \\
& \left.\boldsymbol{\tau}_{h} \boldsymbol{n}_{\omega_{\boldsymbol{a}}}=\Pi_{\boldsymbol{\Sigma}_{h} \boldsymbol{n}_{\omega \boldsymbol{a}}}\left(\psi_{\boldsymbol{a}}\left(\left[P_{1, \gamma}^{n}\left(\boldsymbol{u}_{h}^{k}\right)\right]_{\mathrm{reg}, \delta}-\left[P_{1, \gamma}^{n}\left(\boldsymbol{u}_{h}^{k}\right)\right]_{\mathbb{R}^{-}}\right) \boldsymbol{n}\right) \text { on } \partial \omega_{\boldsymbol{a}} \cap \Gamma_{C}\right\}, \\
& \boldsymbol{\Sigma}_{h, N, C, \text { lin }}^{\boldsymbol{a}, k}:=\left\{\boldsymbol{\tau}_{h} \in \boldsymbol{\Sigma}_{h}\left(\omega_{\boldsymbol{a}}\right): \boldsymbol{\tau}_{h} \boldsymbol{n}_{\omega_{\boldsymbol{a}}}=\mathbf{0} \text { on } \partial \omega_{\boldsymbol{a}} \backslash \partial \Omega \text { and on } \partial \omega_{\boldsymbol{a}} \cap \Gamma_{N}\right. \text {, and } \\
& \left.\boldsymbol{\tau}_{h} \boldsymbol{n}_{\omega_{\boldsymbol{a}}}=\Pi_{\boldsymbol{\Sigma}_{h} \boldsymbol{n}_{\omega_{\boldsymbol{a}}}}\left(\psi_{\boldsymbol{a}}\left(P_{\mathrm{lin}}^{k-1}\left(\boldsymbol{u}_{h}^{k}\right)-\left[P_{1, \gamma}^{n}\left(\boldsymbol{u}_{h}^{k}\right)\right]_{\mathrm{reg}, \delta}\right) \boldsymbol{n}\right) \text { on } \partial \omega_{\boldsymbol{a}} \cap \Gamma_{C}\right\},
\end{aligned}
$$

and, for any internal vertex $\boldsymbol{a} \in \mathcal{V}_{h}^{i}, \boldsymbol{\Sigma}_{h, N, C, \bullet}^{\boldsymbol{a}, k}:=\boldsymbol{\Sigma}_{h}^{\boldsymbol{a}}$ (see (5.1)) for $\bullet \in\{$ dis, reg, lin $\}$. Moreover, let $\boldsymbol{y}^{k}, \tilde{\boldsymbol{y}}^{k} \in \boldsymbol{R} \boldsymbol{M}^{d}$ be such that, for all $\boldsymbol{z} \in \boldsymbol{R} \boldsymbol{M}^{d}$,

$$
\begin{aligned}
& \left(\boldsymbol{y}^{k}, z\right)_{\omega_{\boldsymbol{a}}}=\left(-\psi_{\boldsymbol{a}} \boldsymbol{f}+\boldsymbol{\sigma}\left(\boldsymbol{u}_{h}^{k}\right) \boldsymbol{\nabla} \psi_{\boldsymbol{a}}, \boldsymbol{z}\right)_{\omega_{\boldsymbol{a}}}-\left(\Pi_{\boldsymbol{\Sigma}_{h} \boldsymbol{n}_{\omega_{\boldsymbol{a}}}}\left(\psi_{\boldsymbol{a}} \boldsymbol{g}_{N}\right), z\right)_{\partial \omega_{\boldsymbol{a}} \cap \Gamma_{N}} \\
& -\left(\Pi_{\boldsymbol{\Sigma}_{h} \boldsymbol{n}_{\omega \boldsymbol{a}}}\left(\psi_{\boldsymbol{a}}\left[P_{1, \gamma}^{n}\left(\boldsymbol{u}_{h}^{k}\right)\right]_{\mathbb{R}^{-}} \boldsymbol{n}\right), z\right)_{\partial \omega_{\boldsymbol{a}} \cap \Gamma_{C}}, \\
& \left(\tilde{\boldsymbol{y}}^{k}, \boldsymbol{z}\right)_{\omega_{\boldsymbol{a}}}=\left(\Pi_{\boldsymbol{\Sigma}_{h} \boldsymbol{n}_{\omega_{\boldsymbol{a}}}}\left(\psi_{\boldsymbol{a}}\left(\left[P_{1, \gamma}^{n}\left(\boldsymbol{u}_{h}^{k}\right)\right]_{\mathbb{R}^{-}}-\left[P_{1, \gamma}^{n}\left(\boldsymbol{u}_{h}^{k}\right)\right]_{\mathrm{reg}, \delta}\right) \boldsymbol{n}\right), z\right)_{\partial \omega_{\boldsymbol{a}} \cap \Gamma_{C}}
\end{aligned}
$$

if $\boldsymbol{a} \in \mathcal{V}_{h}^{b}$, and $\boldsymbol{y}^{k}=\tilde{\boldsymbol{y}}^{k}=\mathbf{0}$ if $\boldsymbol{a} \in \mathcal{V}_{h}^{i}$. 
Construction 16 (Equilibrated stress reconstruction distinguishing the error components). Let, for - $\in\{$ dis, reg, lin $\}$ and any vertex $\boldsymbol{a} \in \mathcal{V}_{h},\left(\boldsymbol{\sigma}_{h, \bullet}^{\boldsymbol{a}, k}, \boldsymbol{r}_{h, \bullet}^{\boldsymbol{a}, k}, \lambda_{h, \bullet}^{\boldsymbol{a}, k}\right) \in \boldsymbol{\Sigma}_{h, N, C, \bullet}^{\boldsymbol{a}, k} \times \boldsymbol{U}_{h}^{\boldsymbol{a}} \times \boldsymbol{\Lambda}_{h}^{\boldsymbol{a}}$ be the solution to the following problem:

$$
\begin{aligned}
\left(\sigma_{h, \bullet}^{a, k}, \tau_{h}\right)_{\omega_{\boldsymbol{a}}}+\left(\boldsymbol{r}_{h, \bullet}^{\boldsymbol{a}, k}, \operatorname{div} \tau_{h}\right)_{\omega_{\boldsymbol{a}}}+\left(\lambda_{h, \bullet}^{\boldsymbol{a}, k}, \tau_{h}\right)_{\omega_{\boldsymbol{a}}} & =\left(\tau_{h, \bullet}^{\boldsymbol{a}, k}, \tau_{h}\right)_{\omega_{\boldsymbol{a}}} & & \forall \tau_{h} \in \Sigma_{h}^{\boldsymbol{a}}, \\
\left(\operatorname{div} \boldsymbol{\sigma}_{h, \bullet}^{\boldsymbol{a}, k}, v_{h}\right)_{\omega_{\boldsymbol{a}}} & =\left(\boldsymbol{v}_{h, \bullet}^{\boldsymbol{a}, k}, v_{h}\right)_{\omega_{\boldsymbol{a}}} & & \forall \boldsymbol{v}_{h} \in \boldsymbol{U}_{h}^{\boldsymbol{a}}, \\
\left(\boldsymbol{\sigma}_{h, \bullet}^{\boldsymbol{a}, k}, \boldsymbol{\mu}_{h}\right)_{\omega_{\boldsymbol{a}}} & =0 & & \forall \boldsymbol{\mu}_{h} \in \boldsymbol{\Lambda}_{h}^{\boldsymbol{a}},
\end{aligned}
$$

where

$$
\boldsymbol{\tau}_{h, \bullet}^{\boldsymbol{a}, k}:=\left\{\begin{array}{ll}
\psi_{\boldsymbol{a}} \sigma\left(\boldsymbol{u}_{h}^{k}\right) & \text { if } \bullet \text { dis, } \\
0 & \text { if } \bullet \in\{\mathrm{reg}, \operatorname{lin}\},
\end{array} \quad \boldsymbol{v}_{h, \bullet}^{\boldsymbol{a}, k}:= \begin{cases}-\psi_{\boldsymbol{a}} \boldsymbol{f}+\boldsymbol{\sigma}\left(\boldsymbol{u}_{h}^{k}\right) \boldsymbol{\nabla} \psi_{\boldsymbol{a}}-\boldsymbol{y}^{k} & \text { if } \bullet=\mathrm{dis}, \\
-\tilde{\boldsymbol{y}}^{k} & \text { if } \bullet=\mathrm{reg}, \\
\boldsymbol{y}^{k}+\tilde{\boldsymbol{y}}^{k} & \text { if } \bullet=\text { lin. }\end{cases}\right.
$$

Extending $\boldsymbol{\sigma}_{h, \bullet}^{\boldsymbol{a}, k}$ by zero outside the patch $\omega_{\boldsymbol{a}}$, we set $\boldsymbol{\sigma}_{h, \bullet}^{k}:=\sum_{\boldsymbol{a} \in \mathcal{V}_{h}} \boldsymbol{\sigma}_{h, \bullet}^{\boldsymbol{a}, k}$, and we define $\boldsymbol{\sigma}_{h}^{k}:=$ $\sigma_{h, \text { dis }}^{k}+\sigma_{h, \text { reg }}^{k}+\sigma_{h, \text { in }}^{k}$

By definition, $\boldsymbol{y}^{k}$ and $\tilde{\boldsymbol{y}}^{k}$ ensure that the forcing terms $\boldsymbol{v}_{h, \boldsymbol{\bullet}}^{\boldsymbol{a}, k}$ satisfy the following Neumann compatibility conditions for $a \in \mathcal{V}_{h}^{b} \backslash \mathcal{V}_{h}^{D}$ :

$$
\begin{gathered}
\left(\boldsymbol{v}_{h, \mathrm{dis}}^{\boldsymbol{a}, k}, z\right)_{\omega_{\boldsymbol{a}}}=\left(\Pi_{\boldsymbol{\Sigma}_{h} \boldsymbol{n}_{\omega_{\boldsymbol{a}}}}\left(\psi_{\boldsymbol{a}} \boldsymbol{g}_{N}\right), z\right)_{\partial \omega_{\boldsymbol{a}} \cap \Gamma_{N}}+\left(\Pi_{\boldsymbol{\Sigma}_{h} \boldsymbol{n}_{\omega_{\boldsymbol{a}}}}\left(\psi_{\boldsymbol{a}}\left[P_{1, \gamma}^{n}\left(\boldsymbol{u}_{h}^{k}\right)\right]_{\mathbb{R}^{-}} \boldsymbol{n}\right), z\right)_{\partial \omega_{\boldsymbol{a}} \cap \Gamma_{C}} \\
\left(\boldsymbol{v}_{h, \mathrm{reg}}^{\boldsymbol{a}, k}, z\right)_{\omega_{\boldsymbol{a}}}=\left(\Pi_{\boldsymbol{\Sigma}_{h} \boldsymbol{n}_{\omega_{\boldsymbol{a}}}}\left(\psi_{\boldsymbol{a}}\left(\left[P_{1, \gamma}^{n}\left(\boldsymbol{u}_{h}^{k}\right)\right]_{\mathrm{reg}, \delta}-\left[P_{1, \gamma}^{n}\left(\boldsymbol{u}_{h}^{k}\right)\right]_{\mathbb{R}^{-}}\right) \boldsymbol{n}\right), z\right)_{\partial \omega_{\boldsymbol{a}} \cap \Gamma_{C}} \\
\left(\boldsymbol{v}_{h, \mathrm{lin}}^{\boldsymbol{a}, k}, z\right)_{\omega_{\boldsymbol{a}}}=\left(\Pi_{\boldsymbol{\Sigma}_{h} \boldsymbol{n}_{\omega_{\boldsymbol{a}}}}\left(\psi_{\boldsymbol{a}}\left(P_{\mathrm{lin}}^{k-1}\left(\boldsymbol{u}_{h}^{k}\right)-\left[P_{1, \gamma}^{n}\left(\boldsymbol{u}_{h}^{k}\right)\right]_{\mathrm{reg}, \delta}\right) \boldsymbol{n}\right), z\right)_{\partial \omega_{\boldsymbol{a}} \cap \Gamma_{C}}
\end{gathered}
$$

for any $\boldsymbol{z} \in \boldsymbol{R} \boldsymbol{M}^{d}$. The obtained tensor $\boldsymbol{\sigma}_{h}^{k}$ is an equilibrated stress reconstruction in the sense of Definition 3, and in particular it satisfies the properties stated by the following lemma whose proof is similar to that of Lemma 15 and is therefore omitted for the sake of conciseness.

Lemma 17 (Properties of $\sigma_{h}^{k}$ ). Let $\sigma_{h}^{k}$ be defined by Construction 16. Then

1. $\sigma_{h, \mathrm{dis}}^{k}, \sigma_{h, \mathrm{reg}}^{k}, \sigma_{h, \mathrm{lin}}^{k}, \sigma_{h}^{k} \in \mathbb{H}(\operatorname{div}, \Omega)$;

2. For every $T \in \mathcal{T}_{h}$ and every $\boldsymbol{v}_{T} \in \mathcal{P}^{p-1}(T),\left(\boldsymbol{d i v} \boldsymbol{\sigma}_{h}^{k}+\boldsymbol{f}, \boldsymbol{v}_{T}\right)_{T}=0$;

3. For every $F \in \mathcal{F}_{h}^{N}$ and every $\boldsymbol{v}_{F} \in \mathcal{P}^{p}(F),\left(\boldsymbol{\sigma}_{h}^{k} \boldsymbol{n}, \boldsymbol{v}_{F}\right)_{F}=\left(\boldsymbol{g}_{N}, \boldsymbol{v}_{F}\right)_{F}$;

4. For every $F \in \mathcal{F}_{h}^{C}$ and every $\boldsymbol{v}_{F} \in \mathcal{P}^{p}(F)$,

$$
\begin{gathered}
\left(\boldsymbol{\sigma}_{h, \mathrm{dis}}^{k} \boldsymbol{n}, \boldsymbol{v}_{F}\right)_{F}=\left(\left[P_{1, \gamma}^{n}\left(\boldsymbol{u}_{h}^{k}\right)\right]_{\mathbb{R}^{-}} \boldsymbol{n}, \boldsymbol{v}_{F}\right)_{F}, \\
\left(\boldsymbol{\sigma}_{h, \mathrm{reg}}^{k} \boldsymbol{n}, \boldsymbol{v}_{F}\right)_{F}=\left(\left(\left[P_{1, \gamma}^{n}\left(\boldsymbol{u}_{h}^{k}\right)\right]_{\mathrm{reg}, \delta}-\left[P_{1, \gamma}^{n}\left(\boldsymbol{u}_{h}^{k}\right)\right]_{\mathbb{R}^{-}}\right) \boldsymbol{n}, \boldsymbol{v}_{F}\right)_{F},
\end{gathered}
$$

and

$$
\left(\boldsymbol{\sigma}_{h, \text { lin }}^{k} \boldsymbol{n}, \boldsymbol{v}_{F}\right)_{F}=\left(\left(P_{\operatorname{lin}}^{k-1}\left(\boldsymbol{u}_{h}^{k}\right)-\left[P_{1, \gamma}^{n}\left(\boldsymbol{u}_{h}^{k}\right)\right]_{\mathrm{reg}, \delta}\right) \boldsymbol{n}, \boldsymbol{v}_{F}\right)_{F}
$$



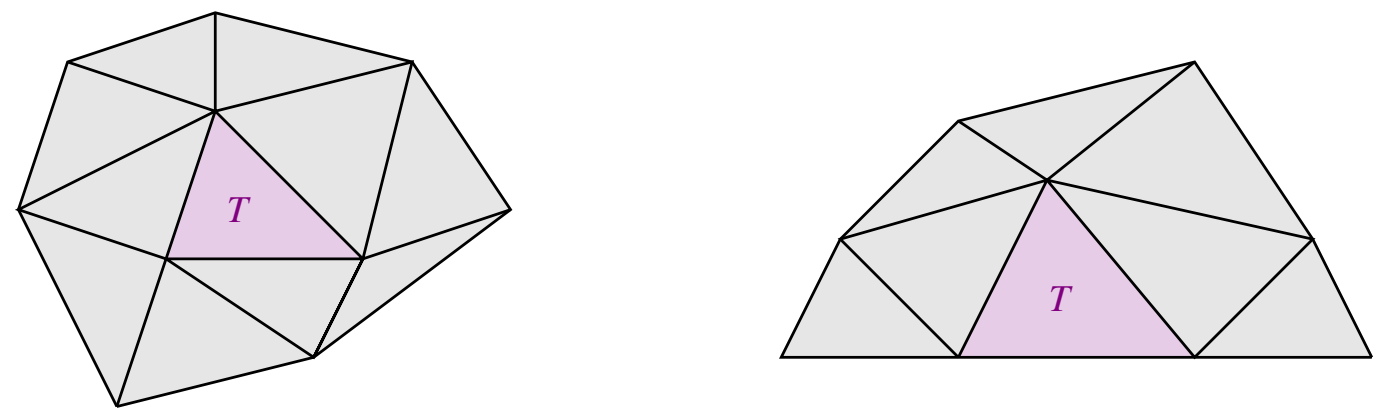

Figure 4: Illustration of $\tilde{\omega}_{T}$ for $T \in \mathcal{T}_{h}$ such that $\mathcal{F}_{T}^{b}=\emptyset($ left $)$ and that $\mathcal{F}_{T}^{b} \neq \emptyset($ right $)$.

Remark 18 (Validity of Property 4. in Definition 3 and of Assumption 10). The fourth property of the previous lemma implies that $\left.\left(\boldsymbol{\sigma}_{h, \boldsymbol{\bullet}}^{k} \boldsymbol{n}\right)\right|_{F}$ has the same direction as the normal vector $\boldsymbol{n}$, and, as a consequence, $\boldsymbol{\sigma}_{h, \bullet}^{k, \boldsymbol{t}}=\mathbf{0}$ on $F \in \mathcal{F}_{h}^{C}$, for $\bullet \in\{$ dis, reg, lin $\}$. Moreover, by definition, $\boldsymbol{\sigma}_{h}^{k}$ is the sum of three tensors representing discretization, regularization and linearization, respectively. Therefore, $\sigma_{h}^{k}$ is an equilibrated stress reconstruction in the sense of Definition 3 that additionally satisfies Assumption 10.

Remark 19 (Alternative expressions of local estimators). Thanks to Lemma 17, we can rewrite the oscillation (4.4a), Neumann (4.4e), and contact (4.4f) estimators as follows:

$$
\begin{gathered}
\eta_{o s c, T}^{k}=\frac{h_{T}}{\pi}\left\|\boldsymbol{f}-\Pi_{T}^{p-1} \boldsymbol{f}\right\|_{T}, \quad \eta_{\text {Neu, },}^{k}=\sum_{F \in \mathcal{F}_{T}^{C}} C_{t, T, F} h_{F}^{1 / 2}\left\|\boldsymbol{g}_{N}-\boldsymbol{\Pi}_{F}^{p} \boldsymbol{g}_{N}\right\|_{F}, \\
\eta_{c n t, T}^{k}=\sum_{F \in \mathcal{F}_{T}^{C}} h_{F}^{1 / 2}\left\|\left[P_{1, \gamma}^{n}\left(\boldsymbol{u}_{h}^{k}\right)\right]_{\mathbb{R}^{-}}-\Pi_{F}^{p}\left[P_{1, \gamma}^{n}\left(\boldsymbol{u}_{h}^{k}\right)\right]_{\mathbb{R}^{-}}\right\|_{F},
\end{gathered}
$$

where $\Pi_{T}^{p-1}, \Pi_{F}^{p}$, and $\Pi_{F}^{p}$ denote the $L^{2}$-orthogonal projectors on the polynomial spaces $\boldsymbol{P}^{p-1}(T)$, $\mathcal{P}^{p}(F)$, and $\mathcal{P}^{p}(F)$, respectively.

\section{Efficiency of local estimators}

In this section we briefly discuss the local efficiency of the estimators defined by (4.4) when using the stress reconstruction described in Section 5.2.

Following [31], for any $T \in \mathcal{T}_{h}$ we denote by $\tilde{\omega}_{T}$ the union of all elements sharing at least one vertex with $T$ (see Figure 4) and by $\mathcal{T}_{T}$ the corresponding set of elements. Moreover, as in Subsection 3.3, $a \lesssim b$ stands for $a \leq C b$, where $C>0$ is a constant which is independent of the mesh size $h$ and of the Nitsche parameter $\gamma_{0}$. We introduce, for all $T \in \mathcal{T}_{h}$, the local residual defined as follows: For all $\boldsymbol{w}_{h} \in \boldsymbol{V}_{h}$ and all $\boldsymbol{v} \in \boldsymbol{H}_{D}^{1}\left(\tilde{\omega}_{T}\right)$

$$
\left\langle\mathcal{R}_{\mathcal{T}_{T}}\left(\boldsymbol{w}_{h}\right), \boldsymbol{v}\right\rangle_{\tilde{\omega}_{T}}:=(\boldsymbol{f}, \boldsymbol{v})_{\tilde{\omega}_{T}}+\left(\boldsymbol{g}_{N}, \boldsymbol{v}\right)_{\partial \tilde{\omega}_{T} \cap \Gamma_{N}}-\left(\boldsymbol{\sigma}\left(\boldsymbol{w}_{h}\right), \boldsymbol{\varepsilon}(\boldsymbol{v})\right)_{\tilde{\omega}_{T}}+\left(\left[P_{1, \gamma}^{n}\left(\boldsymbol{w}_{h}\right)\right]_{\mathbb{R}^{-}}, v^{n}\right)_{\partial \tilde{\omega}_{T} \cap \Gamma_{C}},
$$

where

$$
\boldsymbol{H}_{D}^{1}\left(\tilde{\omega}_{T}\right):=\left\{\boldsymbol{v} \in \boldsymbol{H}^{1}\left(\tilde{\omega}_{T}\right): \boldsymbol{v}=\mathbf{0} \text { on } \partial \tilde{\omega}_{T} \cap \Gamma_{D} \text { and on } \partial \tilde{\omega}_{T} \cap \Omega\right\} .
$$

Letting

$$
\|\boldsymbol{v}\|_{\tilde{\omega}_{T}}:=\left(\|\boldsymbol{\nabla} \boldsymbol{v}\|_{\tilde{\omega}_{T}}^{2}+|\boldsymbol{v}|_{C, \tilde{\omega}_{T}}^{2}\right)^{1 / 2}=\left(\|\boldsymbol{\nabla} \boldsymbol{v}\|_{\tilde{\omega}_{T}}^{2}+\sum_{F \in \mathcal{F}_{T_{T}^{C}}^{C}} \frac{1}{h_{F}}\|\boldsymbol{v}\|_{F}^{2}\right)^{1 / 2},
$$


with $\mathcal{F}_{\mathcal{T}_{T}}^{C}$ denoting the (possibly empty) set of faces of $\mathcal{T}_{T}$ that lie on $\Gamma_{C}$, the corresponding dual norm of the local residual for a function $\boldsymbol{w}_{h} \in \boldsymbol{V}_{h}$ is

$$
\left\|\mathcal{R}_{\mathcal{T}_{T}}\left(\boldsymbol{w}_{h}\right)\right\|_{*, \tilde{\omega}_{T}}=\sup _{\boldsymbol{v} \in \boldsymbol{H}_{D}^{1}\left(\tilde{\omega}_{T}\right),\|\boldsymbol{v}\|_{\tilde{\omega}_{T}=1}}\left\langle\mathcal{R}_{\mathcal{T}_{T}}\left(\boldsymbol{w}_{h}\right), \boldsymbol{v}\right\rangle_{\tilde{\omega}_{T}} .
$$

Theorem 20 (Local efficiency). Assume $d=2$. Let $\boldsymbol{u}_{h}^{k} \in \boldsymbol{V}_{h}$ and let $\boldsymbol{\sigma}_{h}^{k}$ be the stress reconstruction of Construction 16, and assume that the local stopping criteria (4.9) are used in Lines 9 and 11 of Algorithm 1, respectively. Then, for every element $T \in \mathcal{T}_{h}$, it holds

$$
\begin{aligned}
\eta_{\mathrm{osc}, T}^{k}+\eta_{\mathrm{str}, T}^{k}+\eta_{\mathrm{Neu}, T}^{k}+\eta_{\mathrm{cnt}, T}^{k}+\eta_{\mathrm{lin}, T}^{k}+ & \eta_{\mathrm{reg}, T}^{k} \\
& \lesssim\left\|\mathcal{R}_{\mathcal{T}_{T}}\left(\boldsymbol{u}_{h}^{k}\right)\right\|_{*, \tilde{\omega}_{T}}+\eta_{\mathrm{osc}, \mathcal{T}_{T}}^{k}+\eta_{\mathrm{Neu}, \mathcal{T}_{T}}^{k}+\eta_{\mathrm{cnt}, \mathcal{T}_{T}}^{k},
\end{aligned}
$$

where

$$
\eta_{\bullet, \mathcal{T}_{T}}^{k}:=\left(\sum_{T^{\prime} \in \mathcal{T}_{T}}\left(\eta_{\bullet, T^{\prime}}^{k}\right)^{2}\right)^{1 / 2} \quad \text { with } \bullet \in\{\text { osc, Neu, cnt }\} .
$$

Remark 21 (Restriction on the space dimension). The proof of Lemma 23 below requires the introduction of a space with suitable properties that are known only for in dimension $d=2$ (see the definition of the space $\boldsymbol{M}_{h}^{a}$ in [5, Section 4.4]). This assumption reverberates in Theorem 20, whose proof uses Lemma 23.

Proof of Theorem 20. The proof hinges on classical arguments, so we only briefly outline the main ideas and refer to [22, Section 5] for further details.

We introduce, for any element $T \in \mathcal{T}_{h}$, a local residual-based estimator $\eta_{\sharp, T}^{k}$ on the patch $\tilde{\omega}_{T}$, following the path of [31], and then compare it with the local estimators (4.4) and the dual norm of the local residual operator (6.1). In particular, it is possible to prove the following two lemmas by adapting the approach of [20, Appendix A] and [5, Subsection 4.4], respectively:

Lemma 22 (Control of the residual-based estimator $\eta_{\sharp, T}$ ). For any element $T \in \mathcal{T}_{h}$,

$$
\eta_{\sharp, T}^{k} \lesssim\left\|\mathcal{R}_{\mathcal{T}_{T}}\left(\boldsymbol{u}_{h}^{k}\right)\right\|_{*, \tilde{\omega}_{T}}+\eta_{\mathrm{osc}, \mathcal{T}_{T}}^{k}+\eta_{\mathrm{Neu}, \mathcal{T}_{T}}^{k}+\eta_{\mathrm{cnt}, \mathcal{T}_{T}}^{k},
$$

Lemma 23 (Control of the local stress estimator). Assume $d=2$. Then, for every element $T \in \mathcal{T}_{h}$,

$$
\eta_{\mathrm{str}, T}^{k} \lesssim \eta_{\sharp, T}^{k} .
$$

The estimate (6.2) follows using the local stopping criteria along with Lemmas 22 and 23.

Remark 24 (Global efficiency). The results of this subsection can be easily extended in order to prove the global efficiency. Indeed, combining the global criteria shown in Line 9 and 11 of Algorithm 1 and the global counterpart of Lemma 22 and 23, we achieve

$$
\eta_{\text {osc }}^{k}+\eta_{\text {str }}^{k}+\eta_{\text {Neu }}^{k}+\eta_{\text {cnt }}^{k}+\eta_{\text {lin }}^{k}+\eta_{\text {reg }}^{k} \lesssim\left\|\mathcal{R}\left(\boldsymbol{u}_{h}^{k}\right)\right\|_{*}+\eta_{\text {osc }}^{k}+\eta_{\text {Neu }}^{k}+\eta_{\text {cnt }}^{k} .
$$

\section{Numerical results}

We present numerical cases to validate the a posteriori error estimate of Theorem 11 and show its use in the framework of an adaptive algorithm. The simulations are performed with the open 


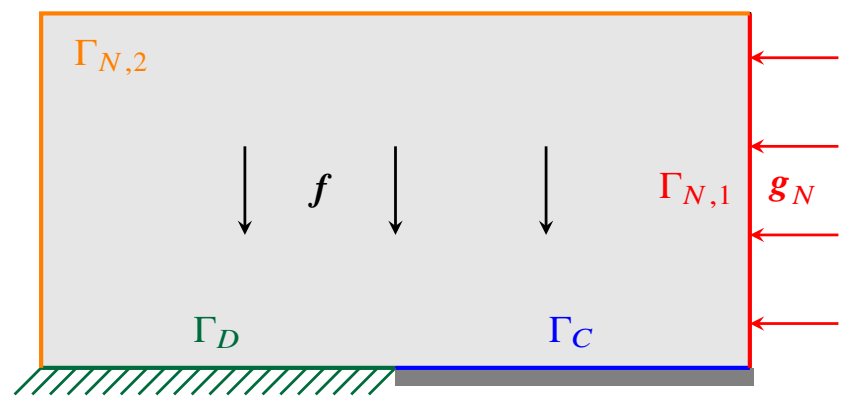

Figure 5: Illustration of the rectangular domain with the subdivision of the boundary as $\partial \Omega=$ $\Gamma_{D} \dot{\cup}\left(\Gamma_{N, 1} \dot{\cup} \Gamma_{N, 2}\right) \dot{\cup} \Gamma_{C}$.
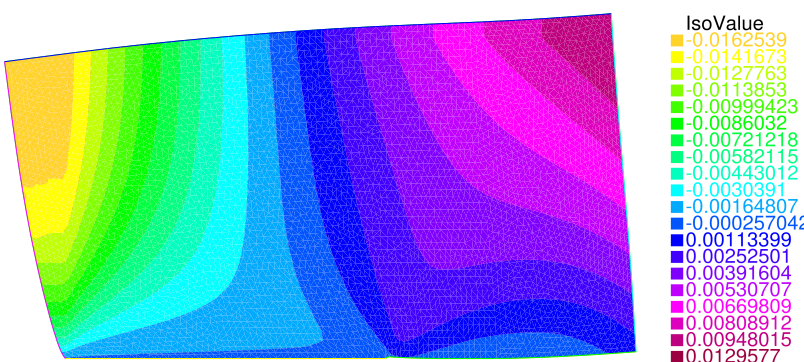

(a) Vertical displacement in the deformed domain (amplification factor $=5$ ).

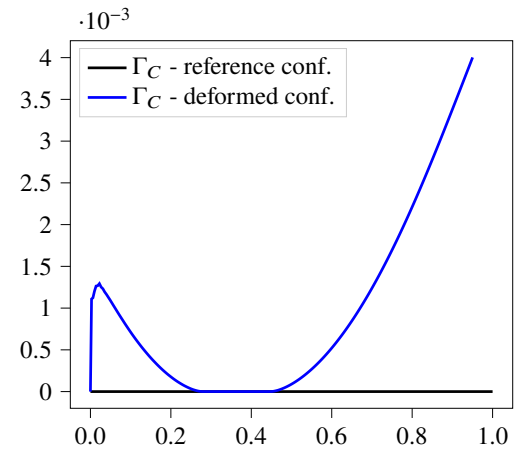

(b) Displacement of the contact boundary.

Figure 6: Vertical displacement in the deformed configuration (left), and representation of the contact boundary part $\Gamma_{C}$ in the reference (black) and deformed (blue) configuration (right).

source finite element library FreeFem++ (see [25] and also https://freefem.org/). We will use the notion of local and global total estimator: $\eta_{\mathrm{tot}, \mathrm{T}}$ defined by (4.8) and

$$
\eta_{\mathrm{tot}}:=\left(\sum_{T \in \mathcal{T}_{h}}\left(\eta_{\mathrm{tot}, T}\right)^{2}\right)^{1 / 2} .
$$

For the sake of brevity, above and throughout this section we omit the superscript $k$ which identifies the step of the Newton method.

We consider a body that, in its reference configuration, occupies the rectangular domain $\Omega=(-1,1) \times(0,1)$ (see Figure 5), with mechanical parameters $E=1$ and $v=0.3$, corresponding to Lamé coefficients $\mu \approx 0.385$ and $\lambda \approx 0.577$. The body is subjected to a weight force $f=$ $(0,-0.01)$. Homogeneous Dirichlet boundary conditions are enforced on $\Gamma_{D}=(-1,0) \times\{0\}$, and the the body in its undeformed configuration is in contact with a rigid horizontal interface on $\Gamma_{C}=(0,1) \times\{0\}$. The Nitsche parameter is $\gamma_{0}=100 E$, whereas the regularization parameter which defines the operator $[\cdot]_{\mathrm{reg}, \delta}$ is $\delta=E / 100$. A pressure $\boldsymbol{g}_{N}=(-0.0275,0)$ acts on the right side of the body $\Gamma_{N, 1}=\{1\} \times(0,1)$, and the rest of the boundary is free, i.e., $\boldsymbol{g}_{N}=\mathbf{0}$ on $\Gamma_{N, 2}=\{-1\} \times(0,1) \cup(-1,1) \times\{1\}$. Since a closed-form solution is not available for this configuration, we take as reference solution the function $\overline{\boldsymbol{u}}_{h}$ computed solving (2.7) with Lagrange $\mathcal{P}^{2}$ finite elements on a fine mesh $(h \approx 0.0084)$. To compute the approximated solution $\boldsymbol{u}_{h}$, we use Lagrange $\mathcal{P}^{1}$ elements (while this choice is known to lock in the quasi-incompressible limit, it is admissible for the set of parameters considered here and is compatible with the use of the lowest-order mixed finite elements available in FreeFem++ to compute the equilibrated stress 

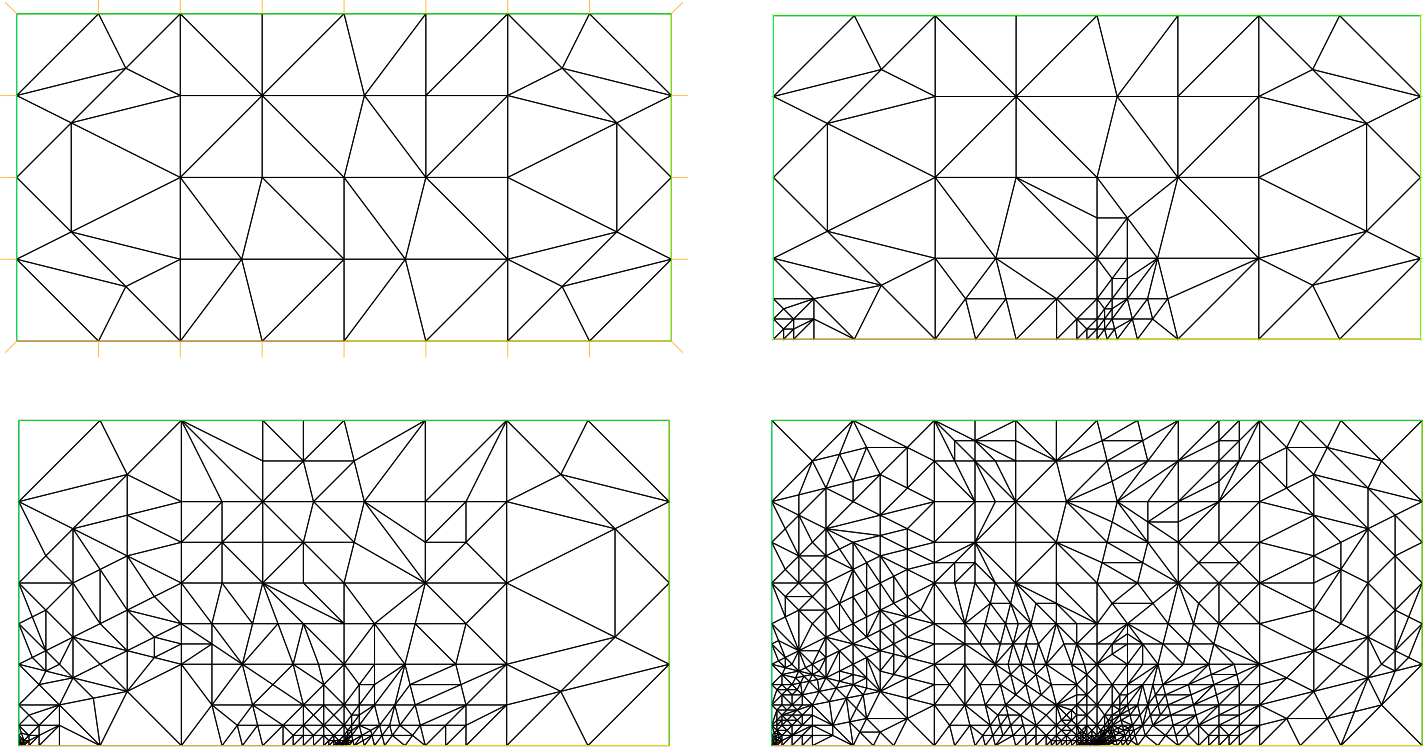

Figure 7: Initial mesh and adaptively refined mesh after 3, 7 and 11 steps, respectively.

\begin{tabular}{c|cccccccccccc}
\hline & Initial & $1^{\text {st }}$ & $2^{\text {nd }}$ & $3^{\text {rd }}$ & $4^{\text {th }}$ & $5^{\text {th }}$ & $6^{\text {th }}$ & $7^{\text {th }}$ & $8^{\text {th }}$ & $9^{\text {th }}$ & $10^{\text {th }}$ & $11^{\text {th }}$ \\
\hline$N_{\text {reg }}$ & 7 & 0 & 1 & 0 & 0 & 0 & 0 & 0 & 0 & 0 & 0 & 0 \\
\hline$N_{\text {lin }}$ & 26 & 2 & 4 & 5 & 3 & 4 & 4 & 4 & 5 & 8 & 8 & 7 \\
\hline
\end{tabular}

Table 2: Number of regularization iterations $N_{\text {reg }}$ and Newton iterations $N_{\text {lin }}$ at each refinement step of the Algorithm 1.

reconstructions). In the deformed configuration, the body is in contact with the rigid foundation in a non-empty interval $I_{C} \subset \Gamma_{C}$ which is approximately $(0.279,0.447)$. Figure 6 a shows the vertical displacement in the deformed domain with an amplification factor equal to 5. Moreover, in Figure 6b, which display the contact boundary part $\Gamma_{C}$ in the reference domain (black) and in the deformed domain (blue), we can easily identify the contact interval $I_{C}$.

We refine adaptively the initial mesh following the distribution of the local total estimator $\eta_{\text {tot }, T}$, refining only the elements in which the value of this estimator is higher. In particular, at each refinement step, the $6 \%$ elements with larger estimated error are refined, i.e., are subdivided into four sub-triangles dividing each edge by two. Figure 7 shows the initial mesh and the result of adaptive refinement after 3, 7, and 11 steps, respectively. We remark that the refinement is concentrated on the endpoints of $\Gamma_{D}$ (singularities due to the homogeneous Dirichlet conditions) and near the contact interval $I_{C}$. Figure 8 a compares the convergence on uniformly and adaptively refined meshes for the $H^{1}$-norm $\left\|\overline{\boldsymbol{u}}_{h}-\boldsymbol{u}_{h}\right\|_{1, \Omega}$ and for the energy norm $\left\|\overline{\boldsymbol{u}}_{h}-\boldsymbol{u}_{h}\right\|_{\mathrm{en}}$, showing the corresponding curves as functions of the number of degrees of freedom. In particular, the uniform refinement is performed dividing all triangles of the mesh into four sub-triangles. The adaptive approach provides better convergence rates, i.e., adopting it we can achieve a fixed level of precision with fewer degrees of freedom. The asymptotic convergence rates are approximately 0.309 and 0.255 in the uniform case, and 0.450 and 0.449 in the adaptive case, for the $H^{1}$-norm and energy norm, respectively (the optimal convergence rate for smooth solutions is 0.5 ).

We recall that the measure of the error is the dual norm $\left\|\mathcal{R}\left(\boldsymbol{u}_{h}\right)\right\|_{*}$ defined by (3.4), which is not computable (it can be, however, approximated through an elliptic lifting). As a consequence, 


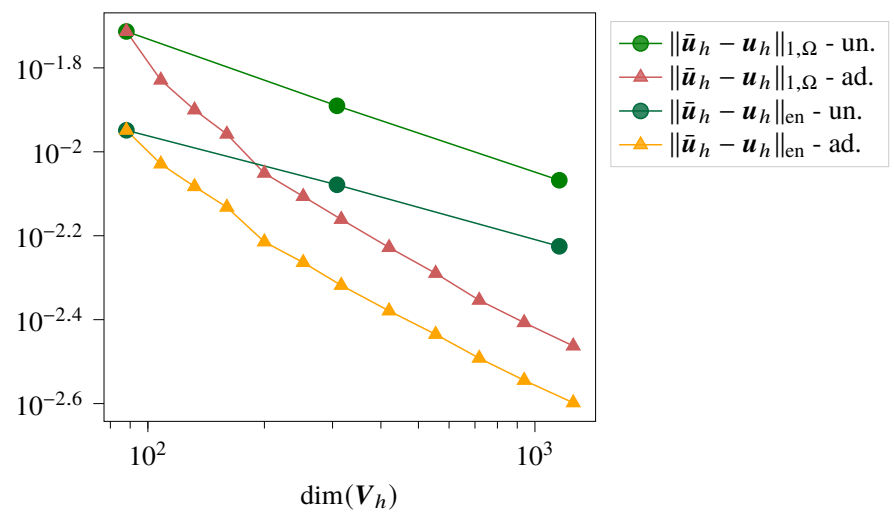

(a) $\left\|\overline{\boldsymbol{u}}_{h}-\boldsymbol{u}_{h}\right\|_{1, \Omega}$ and $\left\|\overline{\boldsymbol{u}}_{h}-\boldsymbol{u}_{h}\right\|_{\text {en }}$.

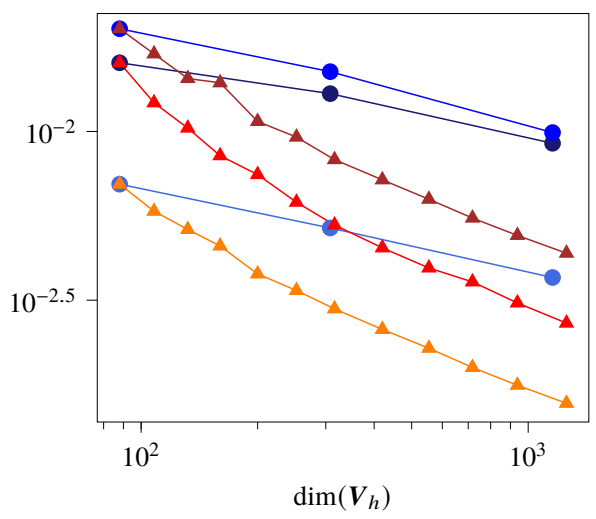

(b) $\eta_{\text {tot }}, \mathcal{L}\left(\boldsymbol{u}_{h}\right)$ and $\mathcal{U}\left(\boldsymbol{u}_{h}\right)$

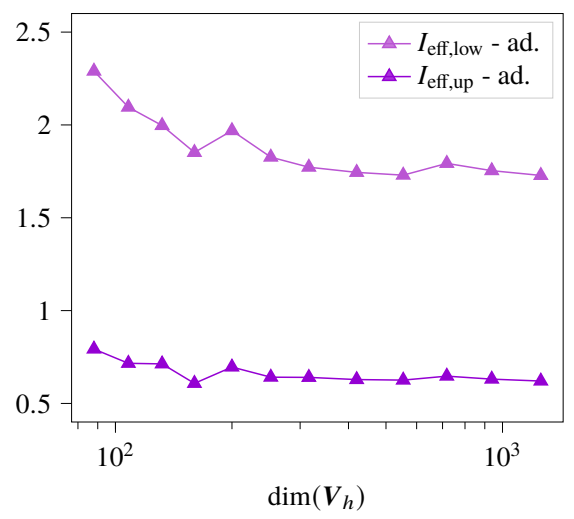

(c) $I_{\text {eff,low }}$ and $I_{\text {eff,up }}$.

Figure 8: Comparison between the uniform case (circles) and the adaptive one (triangle) for the $H^{1}$-norm $\left\|\overline{\boldsymbol{u}}_{h}-\boldsymbol{u}_{h}\right\|_{1, \Omega}$ and the energy norm $\left\|\overline{\boldsymbol{u}}_{h}-\boldsymbol{u}_{h}\right\|_{\mathrm{en}}$ (top), and for the global total estimator $\eta_{\text {tot }}, \mathcal{L}\left(\boldsymbol{u}_{h}\right)$ and $\mathcal{U}\left(\boldsymbol{u}_{h}\right)$ (bottom-left). Corresponding effectivity indices $I_{\text {eff,low }}$ and $I_{\text {eff,up }}$ (bottomright).

recalling Theorems 7 and 9, we compare the global total estimator $\eta_{\text {tot }}$ with the following quantities:

$$
\mathcal{L}\left(\boldsymbol{u}_{h}\right):=\mu^{1 / 2}\left\|\overline{\boldsymbol{u}}_{h}-\boldsymbol{u}_{h}\right\|_{\mathrm{en}}
$$

and

$$
\mathcal{U}\left(\boldsymbol{u}_{h}\right):=(d \lambda+4 \mu)^{1 / 2}\left\|\overline{\boldsymbol{u}}_{h}-\boldsymbol{u}_{h}\right\|_{\mathrm{en}}+\left(\sum_{F \in \mathcal{F}_{h}^{C}} h_{F}\left\|\sigma^{n}\left(\overline{\boldsymbol{u}}_{h}\right)-\left[P_{1, \gamma}^{n}\left(\boldsymbol{u}_{h}\right)\right]_{\mathbb{R}^{-}}\right\|_{F}^{2}\right)^{1 / 2} .
$$

In particular, the latter incorporates an additional error component on the contact interface. Furthermore, we define the two following effectivity indices:

$$
I_{\text {eff,low }}:=\frac{\eta_{\text {tot }}}{\mathcal{L}\left(\boldsymbol{u}_{h}\right)}=\frac{\eta_{\text {tot }}}{\mu^{1 / 2}\left\|\overline{\boldsymbol{u}}_{h}-\boldsymbol{u}_{h}\right\|_{\text {en }}} \quad \text { and } \quad I_{\text {eff,up }}:=\frac{\eta_{\text {tot }}}{\mathcal{U}\left(\boldsymbol{u}_{h}\right)} .
$$

The results are illustrated in Figure $8 \mathrm{~b}$ and $8 \mathrm{c}$. The total estimator always remains between the energy norm rescaled by a Lamé parameter $\mathcal{L}\left(\boldsymbol{u}_{h}\right)$ and the upper bound for the dual residual norm $\mathcal{U}\left(\boldsymbol{u}_{h}\right)$, i.e., $I_{\text {eff,low }}>1$ and $I_{\text {eff,up }}<1$. Figure 9 shows the distribution of the local total estimator 

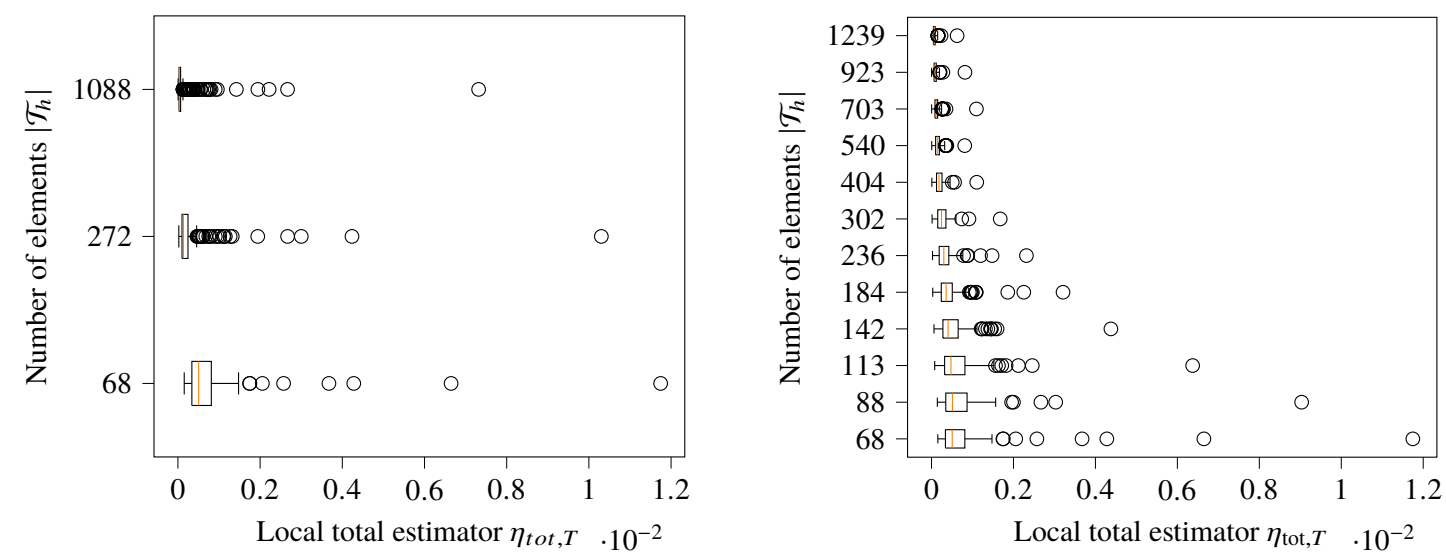

Figure 9: Evolution of the distribution of the local total estimator $\eta_{\mathrm{tot}, T}$ over the mesh with uniform refinement (left) and adaptive refinement (right). The right panel shows that the interval $\left(\min _{T \in \mathcal{T}_{h}} \eta_{\mathrm{tot}, T}, \max _{T \in \mathcal{T}_{h}} \eta_{\text {tot }, T}\right)$ shrinks much faster in the adaptively refined case than in the uniformly refined one. The labels of the $y$-axis report the number of elements of the corresponding mesh.

$\eta_{\text {tot }, T}$ at each mesh refinement step in both the uniform and adaptive frameworks. Here, we use boxplots to see where values concentrate. With the adaptive approach, all the local estimators $\left\{\eta_{\mathrm{tot}, T}\right\}_{T \in \mathcal{T}_{h}}$ are contained in an interval that becomes smaller and smaller at each refinement iteration, and the decrease of the maximum value is significantly faster than in the uniformly refined computation. Indeed, in the latter there is always a value which is much bigger than the others even if the number of degrees of freedom and the number of elements are high (in the last case, $\left|V_{h}\right|=1156$ and $\left|\mathcal{T}_{h}\right|=1088$ ), showing that the error concentrates in specific areas. Figure 10 compares the selection of triangles to refine (highlighted in green) using the distribution of the energy norm $\left\|\overline{\boldsymbol{u}}_{h}-\boldsymbol{u}_{h}\right\|_{\mathrm{en}, \mathrm{T}}$ (left) and the total estimator $\eta_{\mathrm{tot}, \mathrm{T}}$ (right) for the initial mesh and the adaptively refined mesh after 6 and 10 steps. The sets of selected triangles are concentrated in the same zones.

Finally, we apply the fully adaptive Algorithm 1 with $\gamma_{\text {reg }}=0.04$ and $\gamma_{\text {lin }}=0.08$. The initial regularization parameter $\delta$ is taken equal to the Young modulus $E$ and, at each step in which the global stopping criterion shown in Line 11 of Algorithm 1 is not satisfied, we divide it by 2 . Table 2 contains the number of regularization and Newton iterations, denoted by $N_{\text {reg }}$ and $N_{\text {lin }}$ respectively, and Figure 11a displays the curves of the different global estimators for 11 adaptive refinement steps as functions of the degrees of freedom. The same estimators are shown in Figure 11b and $11 \mathrm{c}$ as functions of the Newton iterations for the 3rd and 9th adaptively refined meshes. A circle underlines the step (5th and 8th, respectively) at which the global stopping criterion of Line 9 is reached. At this step, the regularization estimator satisfies the global stopping criterion of Line 11, and the other ones have already stabilized.

\section{Acknowledgements}

This project has received funding from the European Union's Horizon 2020 research and innovation program under grant agreement No 847593. 

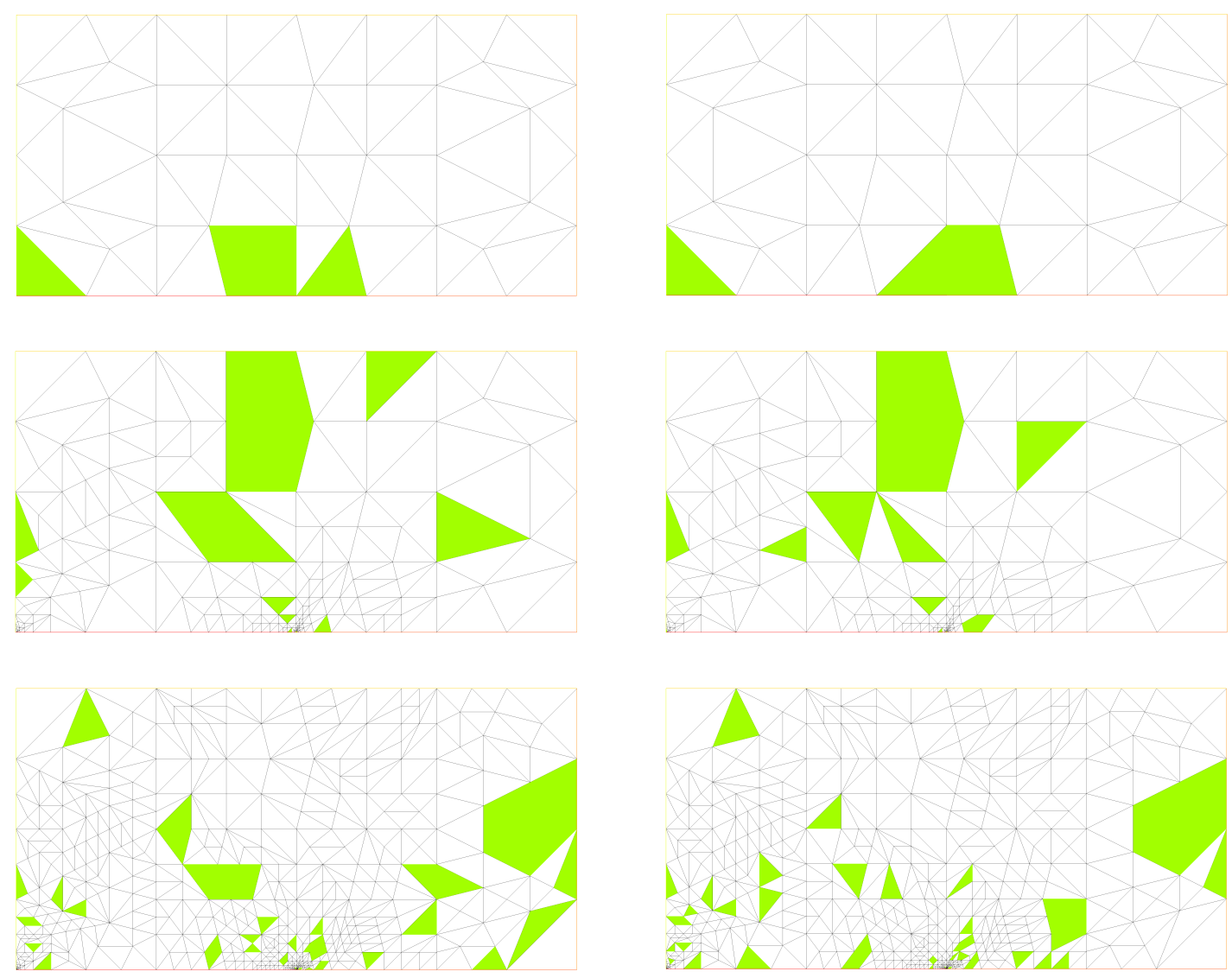

Figure 10: Triangles to refine following the distribution of $\left\|\overline{\boldsymbol{u}}_{h}-\boldsymbol{u}_{h}\right\|_{\text {en }}$ (left) and of $\eta_{\text {tot }}$ (left) for the initial mesh (top), and adaptively refined mesh after 6 and 10 steps (middle and bottom, respectively).

\section{References}

[1] D. N. Arnold, R. S. Falk, And R. Winther, Mixed finite element methods for linear elasticity with weakly imposed symmetry, Mathematics of Computation, 76 (2007), pp. 1699-1723.

[2] D. N. Arnold And R. Winther, Mixed finite elements for elasticity, Numerische Mathematik, 92 (2002), pp. 401-419.

[3] C. Bernardi And V. Girault, A local regularization operator for triangular and quadrilateral finite elements, SIAM Journal of Numerical Analysis, 35 (1998), pp. 1893-1916.

[4] D. Boffi, F. Brezzi, And M. Fortin, Mixed Finite Element Methods and Applications, vol. 44 of Springer Series in Computational Mathematics, Springer Science \& Business Media, 2013.

[5] M. BotTI AND R. RiedLbECK, Equilibrated stress tensor reconstruction and a posteriori error estimation for nonlinear elasticity, Computional Methods in Applied Mathematics, 20 (2020), pp. 39-59.

[6] F. Brezzi And M. Fortin, Mixed and Hybrid Finite Element Methods, vol. 15 of Springer Series in Computational Mathematics, Springer-Verlag, 1991.

[7] F. Chouly, An adaptation of Nitsche's method to the Tresca friction problem, Journal of Mathematical Analysis and Applications, 411 (2014), pp. 329-339.

[8] F. Chouly, A. Ern, And N. Pignet, A Hybrid High-Order discretization combined with Nitsche's method for contact and Tresca friction in small strain elasticity, SIAM Journal on Scientific Computing, 42 (2020), pp. 2300-2324. 


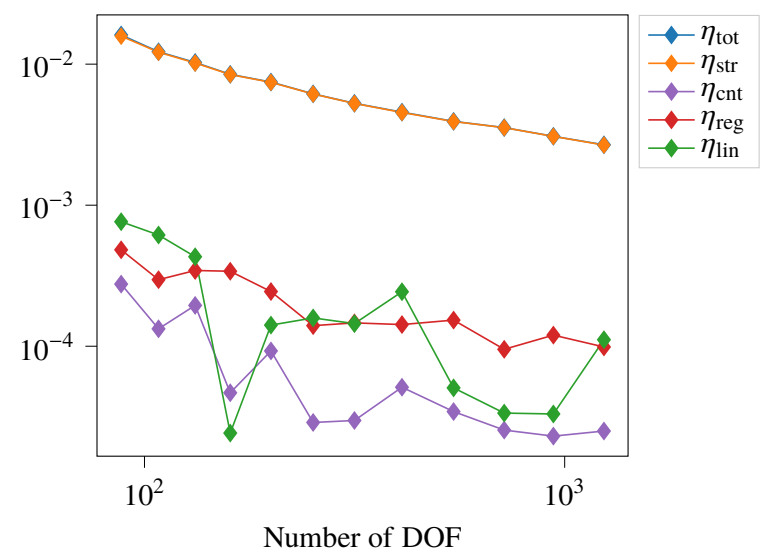

(a) Global estimators with the stopping criteria.

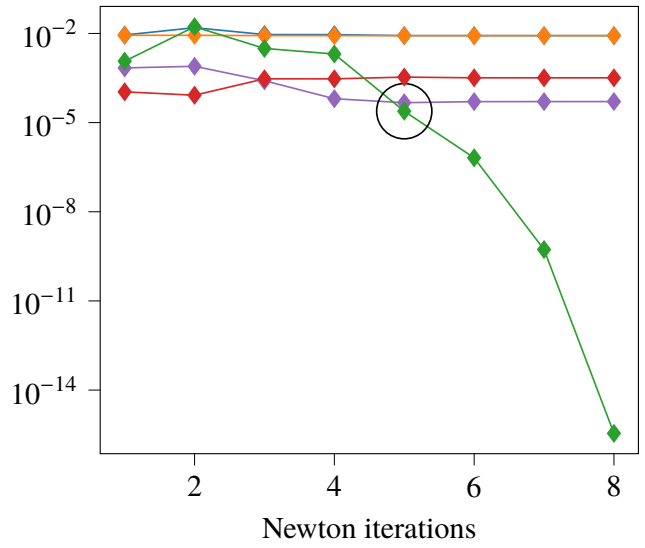

(b) 3rd adaptively refined mesh.

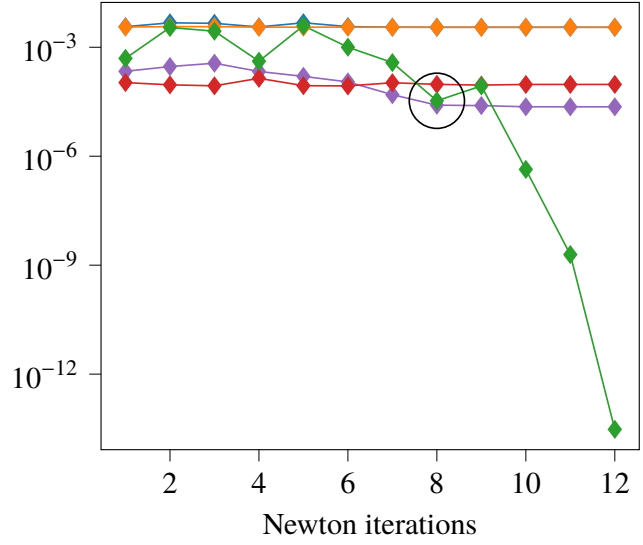

(c) 9 th adaptively refined mesh.

Figure 11: Global estimators $\eta_{\mathrm{tot}}, \eta_{\mathrm{str}}, \eta_{\mathrm{lin}}, \eta_{\mathrm{reg}}$ and $\eta_{\mathrm{cnt}}$ as function of the number of degrees of freedom using the global stopping criteria of Algorithm 1 (top), and as function of Newton iterations for the 3rd and 9th adaptively refined mesh (bottom-left and bottom-right, respectively).

[9] F. Chouly, M. Fabre, P. Hild, R. Mlika, J. Pousin, and Y. Renard, An overview of recent results on Nitsche's method for contact problems, Geometrically Unfitted Finite Element Methods and Applications, 121 (2017), pp. 93-141.

[10] F. Chouly, M. Fabre, P. Hild, J. Pousin, and Y. Renard, Residual-based a posteriori error estimation for contact problems approximated by Nitsche's method, IMA Journal of Numerical Analysis, 38 (2018), pp. 921-954.

[11] F. Chouly And P. Hild, A Nitsche-based method for unilateral contact problems: numerical analysis, SIAM Journal on Numerical Analysis, 51 (2013), pp. 1295-1307.

[12] F. Chouly, P. Hild, And Y. Renard, Symmetric and non-symmetric variants of Nitsche's method for contact problems in elasticity: theory and numerical experiments, Mathematics of Computation, 84 (2015), pp. 1089-1112.

[13] P. G. Ciarlet, The finite element method for elliptic problems, vol. 40 of Classics in Applied Mathematics, Society for Industrial and Applied Mathematics (SIAM), Philadelphia, PA, 2002. Reprint of the 1978 original [North-Holland, Amsterdam; MR0520174 (58 \#25001)].

[14] A. Curnier and P. Alart, A generalized Newton method for contact problems with friction, Journal de Mécanique Théorique et Appliquée, 7 (1988), pp. 67-82. 
[15] D. A. Di Pietro And J. Droniou, The Hybrid High-Order method for polytopal meshes, vol. 19 of Modeling, Simulation and Application, Springer International Publishing, 2020.

[16] D. A. Di Pietro And A. Ern, Mathematical aspects of discontinuous Galerkin methods, vol. 69 of Mathématiques \& Applications (Berlin) [Mathematics \& Applications], Springer, Heidelberg, 2012.

[17] _ A hybrid high-order locking-free method for linear elasticity on general meshes, Comput. Meth. Appl. Mech. Engrg., 283 (2015), pp. 1-21.

[18] D. A. Di Pietro, A. Ern, And S. Lemaire, An arbitrary-order and compact-stencil discretization of diffusion on general meshes based on local reconstruction operators, Comput. Meth. Appl. Math., 14 (2014), pp. 461-472.

[19] D. A. Di Pietro, M. Vohralík, and S. Yousef, Adaptive regularization, linearization, and discretization and a posteriori error control for the two-phase Stefan problem, Mathematics of Computation, 84 (2015), pp. 153-186.

[20] L. El Alaoui, A. ERn, And M. Vohralík, Guaranteed and robust a posteriori error estimates and balancing discretization and linearization errors for monotone nonlinear problems, Computer Methods in Applied Mechanics and Engineering, 200 (2011), pp. 2782-2795.

[21] A. Ern and M. Vohralík, Polynomial-degree-robust a posteriori estimates in a unified setting for conforming, nonconforming, discontinuous galerkin, and mixed discretizations, SIAM Journal on Numerical Analysis, 53 (2015), pp. 1058-1081.

[22] I. Fontana, Modèles d'interface pour les ouvrages hydrauliques, $\mathrm{PhD}$ thesis, Université de Montpellier, École doctorale Information Structures Systèmes, In preparation, 2022.

[23] T. Gustafsson, R. Stenberg, and J. Videman, On Nitsche's method for elastic contact problems, SIAM Journal on Scientific Computing, 42 (2020), pp. B425-B446.

[24] J. Haslinger, I. Hlaváčex, and J. NeČas, Numerical methods for unilateral problems in solid mechanics, in Finite Element Methods (Part 2), Numerical Methods for Solids (Part2), vol. 4 of Handbook of Numerical Analysis, Elsevier, 1996, pp. 313-485.

[25] F. Неснт, New development in FreeFem++, J. Numer. Math., 20 (2012), pp. 251-265.

[26] P. Jiránek, Z. Strakoš, And M. Vohralík, A posteriori error estimates including algebraic error and stopping criteria for iterative solvers, SIAM Journal on Scientific Computing, 32 (2010), pp. 15671590.

[27] J. Niтsche, Über ein variationsprinzip zur lösung von Dirichlet-problemen bei verwendung von teilräumen, die keinen randbedingungen unterworfen sind, Abhandlungen aus dem Mathematischen Seminar der Universität Hamburg, 36 (1971), pp. 9-15.

[28] W. Prager and J. L. Synge, Approximations in elasticity based on the concept of function space, Quart. Appl. Math., 5 (1947), pp. 241-269.

[29] Y. RenARD, Generalized Newton's methods for the approximation and resolution of frictional contact problems in elasticity, Computer Methods in Applied Mechanics and Engineering, 256 (2013), pp. 3855.

[30] R. Riedlbeck, D. A. Di Pietro, And A. ERn, Equilibrated stress tensor reconstructions for linear elasticity problems with application to a posteriori error analysis, in Finite Volumes for Complex Applications VIII - Methods and Theoretical Aspects, 2017, pp. 293-301.

[31] R. VERfüRTH, A review of a posteriori error estimation techniques for elasticity problems, Computer Methods in Applied Mechanics and Engineering, 176 (1999), pp. 419-440.

[32] M. Vohralík, A posteriori error estimates for efficiency and error control in numerical simulations, UPMC Sorbonne Universités, February 2015.

[33] B. Wohlmuth, Variationally consistent discretization schemes and numerical algorithms for contact problems, Acta Numerica, 20 (2011), pp. 569-734. 